JOURNAL OF THE

AMERICAN MATHEMATICAL SOCIETY

Volume 11, Number 1, January 1998, Pages 41-72

S 0894-0347(98)00254-9

\title{
THE SIZE OF THE FIRST EIGENFUNCTION OF A CONVEX PLANAR DOMAIN
}

\author{
DANIEL GRIESER AND DAVID JERISON
}

\section{§1. INTRODUCTION}

Let $\Omega$ be a bounded convex domain in $\mathbf{R}^{2}$. Let $\lambda$ be the lowest eigenvalue of the Laplacian on $\Omega$ with Dirichlet boundary conditions and $u$ the corresponding eigenfunction, that is,

$$
(\Delta+\lambda) u=0 \text { on } \Omega, \quad u=0 \text { on } \partial \Omega .
$$

The goal of this paper is to estimate the size of the first eigenfunction $u$ uniformly for all convex domains. In particular, we will locate the place where $u$ achieves its maximum to within a distance comparable to the inradius, uniformly for arbitrarily large diameter. In addition, we will estimate the location of other level sets of $u$ by showing that $u$ is well-approximated by the first eigenfunction of a naturally associated ordinary differential (Schrödinger) operator. We intend to show in a separate paper that the estimates here are best possible in order of magnitude. The present paper depends on the ideas and results of our earlier work $[\mathrm{J}]$ and [GJ], where detailed estimates for the zero set of the second eigenfunction (or first nodal line) are obtained. The paper $[\mathrm{J}]$ also contains some estimates for the first eigenfunction and lowest eigenvalue, but the techniques of [GJ] and new techniques introduced here are essential to the best possible estimates for the first eigenfunction presented here.

The maximum of the first eigenfunction occurs at the point of largest displacement of a vibrating drum with fixed edges when it vibrates at its fundamental or first resonant frequency. The first nodal line is the stationary curve of the drum at the second resonant frequency. The maximum is harder to find experimentally than the nodal line because it is a single point. Its location has less influence on the eigenvalue or Dirichlet integral, so it is also harder to locate mathematically. Another way to describe the difficulty is as follows. To find the maximum of the first eigenfunction we will need to estimate its first directional derivative. Derivatives of the first eigenfunction are, roughly speaking, analogous to the second eigenfunction because they are solutions to an eigenfunction equation. Moreover, convexity properties of the first eigenfunction imply that the zero set of the derivative divides the region into two connected components. But the derivatives are harder to estimate than a second eigenfunction because they do not vanish at the boundary.

Received by the editors February 17, 1997.

1991 Mathematics Subject Classification. Primary 35J25, 35B65; Secondary 35J05.

Key words and phrases. Convex domains, eigenfunctions.

The first author was a member of the Mathematical Sciences Research Institute, Berkeley. The second author was partially supported by NSF grants DMS-9401355 and DMS-9705825. 
Before defining the ordinary differential operator associated to $\Omega$, rotate $\Omega$ so that the projection onto the $y$-axis has least length and dilate so that the length is 1. Thus we may write

$$
\Omega=\left\{(x, y): f_{1}(x)<y<f_{2}(x), a<x<b\right\}
$$

and the functions $f_{1}$ and $f_{2}$ are convex and concave respectively and satisfy

$$
\begin{gathered}
0 \leq f_{1}(x) \leq f_{2}(x) \leq 1 \quad \text { for } \quad a \leq x \leq b, \\
\max _{[a, b]} f_{2}=1 ; \quad \min _{[a, b]} f_{1}=0 .
\end{gathered}
$$

Note that no further smoothness assumptions are made on $f_{1}$ and $f_{2}$. Let

$$
h(x)=f_{2}(x)-f_{1}(x)
$$

be the height of $\Omega$ at $x$. The relevant Schrödinger operator is

$$
\mathcal{L}=\frac{d^{2}}{d x^{2}}-\frac{\pi^{2}}{h(x)^{2}}
$$

on the interval $[a, b]$, with zero boundary conditions. (See [CL, Chapters 7-9] for a discussion of self-adjoint boundary problems for second-order ordinary differential equations.)

Let $\mu$ be the lowest eigenvalue of $\mathcal{L}$ and let $\phi$ be the first eigenfunction, i.e.,

$$
(\mathcal{L}+\mu) \phi=0 \text { on }(a, b), \quad \phi(a)=\phi(b)=0 .
$$

See Figure 1. The significance of $\mathcal{L}$ is that $\phi(x)$ is the factor depending on $x$ alone in an approximate representation of $u$ using the method of separation of variables.

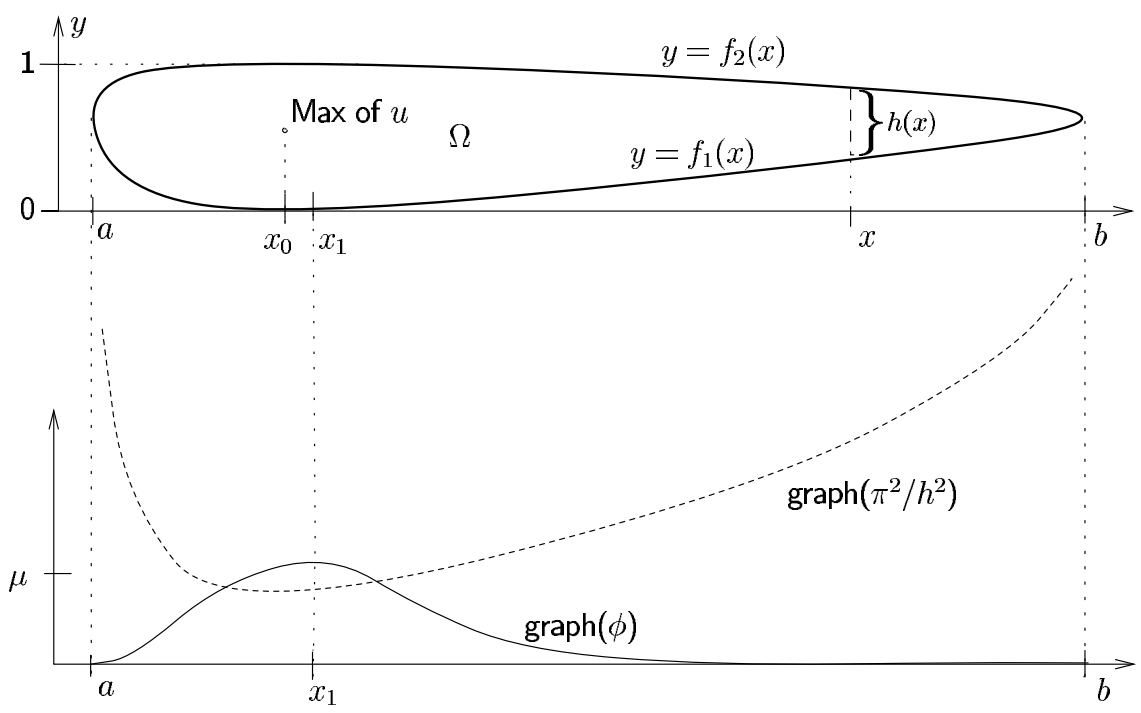

Figure 1: Theorem 1.3 
In the case in which $\Omega$ is a rectangle the representation is exact: $h$ is constant, $\lambda=\mu$, and

$$
u(x, y)=\phi(x) \sin \pi y=\sin (\pi(x-a) / N) \sin \pi y \quad(N=b-a) .
$$

Notice that in order for $u$ to have this product representation it is essential to rotate so that the projection on the $y$ axis has least length. The point is that for arbitrary $\Omega$, this choice of orientation of the axes gives boundary functions $f_{1}(x)$ and $f_{2}(x)$ that are close to constant for large $N$. (The precise size of $f_{i}^{\prime}(x)$ will play an important role in proving the best approximations.) As far as we know, there is only one other type of bounded planar domain for which an exact solution can be expressed in terms of special functions using separation of variables, namely the circular sector. In that case, the solution $\phi$ is an approximation to the (radial) Bessel function in the separation of variables solution in polar coordinates to (1.1). The circular level sets of the Bessel function tend to the vertical level sets of $\phi$, and the approximation gets better and better as $N$ tends to infinity. The theorems of $[\mathrm{J}]$ and $[\mathrm{GJ}]$ and the present work express the extent to which a rectangular, approximate separation of variables works in general.

After multiplication by a constant, we may assume that $u$ and $\phi$ are positive. A theorem of Brascamp and Lieb [BL] implies that the level sets of $u$ are convex. Since $u$ is real analytic in $\Omega$, it follows that $u$ achieves its maximum at exactly one point. The concavity of $h$ implies that $\phi$ has a unique maximum. ${ }^{1}$ The first main theorem of this paper can be stated as follows.

Theorem 1.3. Let $x_{1}$ be such that $\phi\left(x_{1}\right)=\max _{[a, b]} \phi$. Let $\left(x_{0}, y_{0}\right)$ be such that $u\left(x_{0}, y_{0}\right)=\max _{\Omega} u$. There is an absolute constant $C$ such that

$$
\left|x_{0}-x_{1}\right| \leq C
$$

Here we are assuming the unit height normalization of $\Omega$ described above. $C$ is then independent of $\Omega$, but the theorem has no content unless $N>C$. The points $x_{0}$ and $x_{1}$ are depicted in Figure 1.

This theorem should be compared to the analogous theorem for the second eigenfunction:

Theorem B of [J]. Let $u_{2}$ be a second eigenfunction of $\Omega$, and let $\phi_{2}$ be the second eigenfunction of $\mathcal{L}$ on $[a, b]$. There is a unique $x_{2} \in(a, b)$ such that $\phi_{2}\left(x_{2}\right)=0$. If $(x, y) \in \Omega$ is such that $u_{2}(x, y)=0$, then

$$
\left|x-x_{2}\right| \leq C \text {. }
$$

The proof of Theorem 1.3 is based on a detailed comparison of $u$ with $\phi$. To formulate this comparison, we need to recall from $[\mathrm{J}]$ that the essential length scale governing the shape of $u$ is not $N=b-a$, but a number $L$, defined as follows: $L$ is the length of the longest interval $I$ such that (Figure 2; see $\S 3$ )

$$
h(x) \geq 1-\frac{1}{L^{2}} \quad \text { on } \quad I .
$$

\footnotetext{
${ }^{1}$ To prove this, note that $\pi^{2} / h^{2}$ is convex and hence $\phi^{\prime \prime}<0$ on the interval $\left(a^{\prime}, b^{\prime}\right)$ where $\mu-\pi^{2} / h^{2}>0$ and $\phi^{\prime \prime} \geq 0$ on the complement of $\left(a^{\prime}, b^{\prime}\right)$ in $[a, b]$. Since $\phi(a)=\phi(b)=0, \phi$ must be increasing in $\left[a, a^{\prime}\right]$, concave down in $\left(a^{\prime}, b^{\prime}\right)$ and decreasing in $\left[b^{\prime}, b\right]$. Consequently $\phi$ has a unique maximum.
} 
(In what follows the term $-1 / L^{2}$ in $(1.4)$ can be replaced by $-c / L^{2}$ for any fixed $c>0$ with no essential changes.) It is easy to check that

$$
N^{1 / 3} \leq L \leq N .
$$

The two extreme cases are the case of a rectangle, for which $L=N$, and a right triangle, for which $L=N^{1 / 3}$, and, by considering a suitable trapezoid, one sees that any intermediate value for $L$ is possible. The number $L$ is approximately the length of the rectangle inscribed in $\Omega$ of lowest Dirichlet eigenvalue and the projection of that rectangle onto the $x$-axis is comparable to the maximal interval $I$. As shown in $[\mathrm{J}]$, this rectangle is where "most" of the mass of $u$ is concentrated. Roughly speaking $u$ is constant on the central portion of $I$ and decays at least exponentially like $e^{-x / L}$ away from $I$. The same is true for $\phi$.

Denote

$$
\alpha(x, y)=\pi \frac{y-f_{1}(x)}{h(x)} .
$$

The function $\sin \alpha(x, y)$ is one hump of the sine function in the $y$ variable, equal to zero at the upper and lower boundaries, $y=f_{2}(x)$ and $y=f_{1}(x)$. The second main theorem is as follows.

Theorem 1.6. Normalize $u$ and $\phi$ so that $\max u=1$ and $\max \phi=1$. There is an absolute constant $C$ such that

$$
|u(x, y)-\phi(x) \sin \alpha(x, y)| \leq C / L \quad \text { for all } x \in I^{\prime},
$$

where $I^{\prime}$ is the interval concentric with $I$ of half the length.

The interval $I^{\prime}$ can be any fixed proportion of $I$ less than 1 . By adjusting this proportion and the constant in the definition of $I$, one can see that the inequality of Theorem 1.6 holds in any interval on which $\phi(x)\left(\right.$ or $\left.\max _{y} u(x, y)\right)$ is larger than a fixed constant. The proof only gives a constant $C$ that increases as $I^{\prime}$ increases, but we expect that this bound is valid on the whole interval $[a, b]$. In fact, there should be better bounds (perhaps with exponential decay) outside the interval $I^{\prime}$, but we have not carried these out.

The theorems stated here should be contrasted with asymptotic expansions using so-called semiclassical methods. The semiclassical limiting methods allow one to give complete asymptotic expansions as $N \rightarrow \infty$ for eigenfunctions on certain oneparameter families of domains. The bounds proved here are valid uniformly for all domains with the same length scale $L$, and there can be no further terms in an asymptotic formula beyond the first one. Because only the first term in the asymptotics exists, the methods here must be more direct than methods designed for a full asymptotic expansion. On the other hand, the asymptotic expansion can be used on particular examples to show that the theorems here are in the nature of best possible. We plan to carry out this analysis in a separate paper. Consider a domain

$$
\Omega_{1}(N)=\{(x, y): 0 \leq y \leq x, y \leq 1,0 \leq x \leq N\} .
$$

This is a right triangle above $0 \leq x \leq 1$ and a rectangle of width 1 above $1 \leq$ $x \leq N$. Define a second domain $\Omega_{2}$ by symmetrizing $\Omega_{1}$ around the line $y=$ $1 / 2$. Thus the two domains have the same width function $h$ and hence the same function $\phi$. In work in progress, we intend to show that the difference between the locations of the maxima of the first eigenfunctions of the two domains tends 
to a nonzero constant as $N \rightarrow \infty$. (This conjecture is strongly supported by numerical evidence.) This will show that Theorem 1.3 is best possible. Moreover, the difference between the two eigenfunctions is bounded below by $c / N \approx c / L$ for some $c>0$, for example at points of the form $(x, 1 / 2)$ for $N / 8 \leq x \leq N / 4$. More generally, an example using trapezoids modified by a unit segment of slope 1 indicates that there exist eigenfunctions $u$ with the same $\phi$ which differ by $c / L$ no matter what the relationship is between $L$ and $N$. Thus Theorem 1.6 is also best possible.

We will now discuss the relationship between our results and numerical computation. Our results are only meaningful for eccentricities (values of $N$ ) far larger than what is accessible to numerical computation. We make no claims to proving these results with practical bounds on the constants involved. Indeed, there are far too many places where we are not able to give practical bounds, especially in the maximum principle and Harnack inequality aspects of the proof. Nevertheless, numerical examples using the program of Toby Driscoll [D] indicate that the constants can be taken so small that the results give information for essentially all domains. For example, to illustrate Theorem 1.3, when $N>5$, one finds in examples that we believe to be close to the worst possible that the $x$-coordinate of the maximum is within $1 / 5$ of the predicted approximate location $x_{1}$.

This paper and $[\mathrm{J}]$ show that for several questions about eigenfunctions in planar convex domains, a very simple rectangular separation of variables is often adequate. The gain in numerical speed is that one then solves an ordinary differential equation rather than a partial differential equation. This suggests that the same principle works one dimension up. Namely, a separation of variables can, to a certain order of approximation, reduce a three-dimensional partial differential equation to a two-dimensional one, which represents a large computational savings. In higher dimensions, one expects to deal with operators of the form $\Delta+V$, defined on a projection of the convex body, where the potential $V(x)$ (like $\left.\pi^{2} / h(x)^{2}\right)$ is the eigenvalue of the perpendicular cross-section. It is interesting to note that a theorem of [BL] implies that $V(x)^{-1 / 2}$ is a concave function of $x$, just as it is in the onedimensional case. This hypothesis may be useful: It arises in the work of C. Borell [B1], [B2], who proves convexity properties of fundamental solutions associated to Schrödinger operators.

Our theorems treat the noncompact "ends" in the space of all convex domains. Because our results are uniform as the length of the domain tends to infinity (with the width fixed) they can be used as a diagnostic test to see how well a numerical program handles this singularity, in much the way one might look at how well a numerical program handles the graph of an algebraic function near a singularity. For example, the numerical package developed by Toby Driscoll succeeds in the sense that it remains accurate as the domain gets very long and thin. Driscoll computes eigenfunctions of polygonal regions, using, in essence, a polar coordinate expansion at each vertex. Our theoretical results also indicate that for a certain level of accuracy, one can afford to take a polygonal approximation to a region. Moreover, one can even omit certain vertices of a polygon to make it simpler. For estimates of the nodal set to an accuracy on the scale of the inradius, one only needs to sample the width function $h$ at points a distance the inradius apart.

One starting place for connecting this work with the large literature on eigenfunctions is the parameter $L$. This parameter is defined purely geometrically and gives order-of-magnitude information about the eigenfunctions, such as the ratio 
between the $L^{2}$ and $L^{\infty}$ norms. For related work in higher dimensions, see [K]. The earliest instance we know of analysis of a general convex domain using an associated ordinary differential equation is [PW1]. That article proves a sharp lower bound for the lowest (nonzero) Neumann eigenvalue in terms of diameter. The ordinary differential equation of [PW1] is used in [J1] for estimates of the nodal line of the first nontrivial Neumann eigenfunction. Other geometric estimates for eigenvalues and relationships between the first eigenfunction and the heat kernel with applications to probability are investigated in $[\mathrm{KP}],[\mathrm{S}]$, where further references may be found.

We thank Toby Driscoll for computing several examples that indicate that our asymptotics are relevant even for small values of $N$. We thank the referee for many corrections and suggestions.

\section{§2. Main ideas of the PRoOF}

We will compare $u(x, y)$ to the function $\phi(x) e(x, y)$, where $e$ is the $L^{2}$-normalized sine hump in the $y$-direction, that is, with $\alpha$ defined in (1.5),

$$
e(x, y)=\sqrt{2 / h(x)} \sin \alpha(x, y) .
$$

Define

$$
\psi(x)=\int_{f_{1}(x)}^{f_{2}(x)} e(x, y) u(x, y) d y .
$$

The basic idea is that, as motivated by the cases of the rectangle and the circular sector, the function $u$ should be well approximated by its lowest Fourier mode $\psi(x) e(x, y)$ in the $y$-direction, computed for each fixed $x$, and that $\psi$ and $\phi$ satisfy similar ordinary differential equations. We will prove Theorem 1.6 by making the following two estimates.

Theorem 2.1. (a) $|u-\psi e|<C / L^{3}$ on $I^{\prime}$.

(b) $|\psi-\phi / \sqrt{2}|<C / L$ on $I^{\prime}$.

(We do not need the factor $\sqrt{h}$ in (b) because $1-h=O\left(1 / L^{2}\right)$ in $I^{\prime}$.)

Estimate (a) is relatively easy to prove using the main idea of [GJ]. Denote $v=u-\psi e$. Consider the auxiliary function

$$
\tau(x)=\int_{f_{1}(x)}^{f_{2}(x)} v(x, y)^{2} d y .
$$

One can compute that $v$ nearly satisfies the equation $(\Delta+\lambda) v=0$, and hence that $\tau$ satisfies a differential inequality of the form

$$
\tau^{\prime \prime} \geq c \tau-\beta \sqrt{\tau}
$$

for some constant $c$ and measure $\beta \geq 0$. (This convexity inequality for $\tau$ has to be interpreted in the sense of distributions, since the term $\beta$ may have point masses coming from the second derivatives of the boundary $f_{1}$ and $f_{2}$.) The main point is that $c \geq 1$ and $\beta$ is suitably small. The reason why $c \geq 1$ is that $v$ is orthogonal to the first Fourier mode in $y$, and the contributions from higher modes have at least double the eigenvalue. If $\beta$ were zero, $\tau$ could be majorized by a hyperbolic cosine, and this would imply that its values in $I^{\prime}$ would be exponentially small compared to its values far from $I^{\prime}$. In fact, the average of $\beta$ over unit intervals is $O\left(1 / L^{3}\right)$ 
near $I^{\prime}$. And this implies the bound $\tau=O\left(1 / L^{6}\right)$, which, in turn, implies estimate (a). (See Section 5.)

Estimate (a) says that $u$ resembles a sine hump in the vertical direction, but it says nothing about the behavior in the $x$ variable since it merely compares $u$ to its own average. For behavior in the $x$ variable we need the second estimate.

To prove (b) requires much more work. Recall that $(\mathcal{L}+\mu) \phi=0$. We also have

$$
(\mathcal{L}+\mu) \psi=\sigma
$$

where $\sigma$ is an error term estimated (in essence) by

$$
|\sigma| \leq C \int\left|h^{\prime}\right|\left|u_{x}\right| d y+C\left(\frac{\left|h^{\prime}\right|}{L}+\left|h^{\prime}\right|^{2}+\frac{1}{L^{3}}+\left|h^{\prime \prime}\right|\right) \psi .
$$

This estimate follows from the eigenvalue estimate $\lambda-\mu=O\left(1 / L^{3}\right)$ of $[\mathrm{J}]$ and a routine computation. From this, it is not hard to see that the average of $|\sigma|$ over unit intervals in $I^{\prime}$ is $O\left(1 / L^{3}\right)$. But just knowing that $\phi$ and $\psi$ satisfy nearly the same second-order equation is not enough. For example, any translate of the sine function satisfies the same second-order equation. One needs to make use of the boundary conditions $\phi(a)=\phi(b)=0$, which necessitates estimates for $\sigma$ on a much longer interval than $I^{\prime}$. The first step is to show that the functions $\phi$ and $\psi$ are negligibly small where $h<1 / 2$ and also within a unit distance of $a$ or $b$. Call the complement of this region $[\bar{a}, \bar{b}]$. This is the interval on which we must estimate $\sigma$.

The main difficulty then is that the bound for $\sigma$ still refers to $u_{x}$. This means that we have not yet reduced the problem to one concerning ordinary rather than partial differential equations. The natural goal (motivated by the examples of the rectangle and the sector) is that $u_{x}=O(1 / L)$. This is achieved by comparing $u_{x}$ to $\psi^{\prime}$. In fact,

$$
\psi^{\prime}=\int u_{x} e d y+\int u e_{x} d y
$$

and the second term is easy to control. Thus $\psi^{\prime}$ is comparable to an average of $u_{x}$. Unfortunately $u_{x}$ can change sign on vertical lines. Moreover the best estimate for the full gradient $|\nabla u|$ is $O(1) .^{2}$ But one additional feature of $u$ rescues us, namely the fact that $u$ has convex level sets. Crude bounds on the shape of the level set $\{u=1 / 2\}$ (Lemmas 3.16 and 3.17) show that it contains a rectangle of length comparable to $L$. So along "most" of its top and bottom, the slope is bounded by $O(1 / L)$. Since the full gradient $|\nabla u|=O(1)$, it follows that $u_{x}=O(1 / L)$. At the ends of the level set $\{u=1 / 2\}$, the slope is far from small, in fact the tangent lines become vertical, but there are still one-sided bounds of the form $u_{x} \leq C / L$ to the right and $u_{x} \geq-C / L$ to the left. (See Lemma 4.3 and Figure 3.) The one-sided bound combined with control on the average given by $\psi^{\prime}$ gives the control of the form

$$
\left|u_{x}\right| \leq C\left(\left|\psi^{\prime}\right|+(1+\psi) / L\right)
$$

(See Lemma 4.4; the fact that an average of $\left|u_{x}\right|$ controls its supremum is proved in the appendix.) The bounds on $\psi^{\prime}, u_{x}$ and $|\sigma|$ are intertwined: Estimates (2.4)-(2.7) are combined to prove simultaneously the $O(1 / L)$ bound on $u_{x}, \psi^{\prime}$, and the total mass of $|\sigma|$ on $[\bar{a}, \bar{b}]$ (Lemma 4.7).

\footnotetext{
${ }^{2}$ That this is the best one can do is immediate from the $\sin y$ factor appearing in the example of a rectangle. The fact that this bound is true for a general convex domain is proved in Lemma 3.2 .
} 
To deduce Theorem 2.1(b), choose a constant $c_{0}$ such that $\psi$ and $c_{0} \phi$ match at some point. Then the preceding estimates give (see Section 6)

$$
\left|\psi-c_{0} \phi\right| \leq C / L \quad \text { on } \quad I^{\prime} .
$$

The normalization $\max u=1$ and $\max \phi=1$ together with part (a) of Theorem 2.1 then imply that $c_{0}$ can be replaced by $1 / \sqrt{2}$. Thus Theorem 2.1 and hence Theorem 1.6 are proved.

One cannot deduce directly from Theorem 1.6 the location of the maximum of $u$ to the precision given by Theorem 1.3. This is because $\psi$ and $\phi$ only change by the order $O\left(1 / L^{2}\right)$ within a unit distance of the maximum, as the example $\sin (x / L)$ near $x=\pi L / 2$ shows. But one can get around this by proving best possible estimates for the first derivatives of $\psi$ and $\phi$. (The argument is not difficult; it only involves ordinary differential equations. See Section 7.) From Theorem 2.1, one can deduce the following:

$$
\left|\psi^{\prime}-\phi^{\prime} / \sqrt{2}\right| \leq C / L^{2} \quad \text { on } \quad I^{\prime}
$$

Furthermore, if $\phi$ attains its maximum at $x=x_{1}$, then

$$
\left|\phi^{\prime}(x)\right| \geq c\left|x-x_{1}\right| / L^{2} \quad \text { on } \quad I^{\prime}
$$

(Here $I^{\prime}$ must be suitably enlarged so that $x_{1}$ is well inside $I^{\prime}$.) From these two bounds one easily deduces the estimate on the maximum, Theorem 1.3.

\section{§3. Preliminary estimates}

First, we note a basic geometric fact, which will allow us to express any estimates involving the slope of the boundary in terms of $h^{\prime}$ alone:

Lemma 3.1. If the projection of $\Omega$ on the $y$-axis is shortest, then

$$
\left|h^{\prime}(x)\right|=\left|f_{2}^{\prime}(x)\right|+\left|f_{1}^{\prime}(x)\right|, \quad \text { almost everywhere, }
$$

i.e., $f_{1}^{\prime}$ and $f_{2}^{\prime}$ cannot be simultaneously positive or negative.

Proof. Fix $x_{1}$ and denote $s_{1}=f_{1}^{\prime}\left(x_{1}\right), s_{2}=f_{2}^{\prime}\left(x_{1}\right)$. We will show that $s_{2}>s_{1}>0$ leads to a contradiction. (More generally, if the derivative does not exist, $s_{1}$ and $s_{2}$ are defined as the slopes of any tangent lines to $\partial \Omega$ through $\left(x_{1}, f_{1}\left(x_{1}\right)\right)$ and $\left(x_{1}, f_{2}\left(x_{1}\right)\right)$, respectively.) The symmetries $x \rightarrow-x$ and $y \rightarrow-y$ take care of the other three orderings. Thus, in all cases, $s_{1} s_{2}>0$ leads to a contradiction.

Because $\Omega$ is convex, for every $(x, y) \in \Omega$,

$$
\left(x-x_{1}\right) s_{1} \leq f_{1}\left(x_{1}\right)+\left(x-x_{1}\right) s_{1}<y<f_{2}\left(x_{1}\right)+\left(x-x_{1}\right) s_{2} \leq 1+\left(x-x_{1}\right) s_{2} .
$$

The assumption $s_{2}>s_{1}>0$ implies that for $x \leq x_{1}$,

$$
1+\left(x-x_{1}\right) s_{2} \leq 1+\left(x-x_{1}\right) s_{1} .
$$

On the other hand, for $x>x_{1}$

$$
y<1<1+\left(x-x_{1}\right) s_{1} .
$$

Thus for all $x, y<1+\left(x-x_{1}\right) s_{1}$. Combined with the lower bound above,

$$
\left(x-x_{1}\right) s_{1}<y<1+\left(x-x_{1}\right) s_{1} \text {. }
$$

This can be rewritten as

$$
-x_{1} s_{1}<-x s_{1}+y<1-x_{1} s_{1} .
$$


In other words, the points $(x, y) \cdot\left(-s_{1}, 1\right)$ span a segment of length at most 1 . Therefore, the projection of $\Omega$ onto the direction $\left(-s_{1}, 1\right)$ has length at most $1 / \sqrt{s_{1}^{2}+1}$. This contradicts the fact that the shortest projection has length 1 , concluding the proof of the lemma.

In the next lemma, we collect some local estimates:

Lemma 3.2. (a) $\psi(x) \leq h(x)^{1 / 2} \max _{y} u(x, y)$.

(b) There is an absolute constant $C$ such that for $x \in[a+1, b-1]$,

$$
h(x)^{1 / 2} \max _{y} u(x, y) \leq C \psi(x) .
$$

(c) If, in addition, $h(x)>1 / 10$, then

$$
\max _{y}|\nabla u(x, y)| \leq C \max _{y} u(x, y) .
$$

Proof. Part (a) follows from the definition of $\psi$ and from $\int e d y=h(x)^{1 / 2} \sqrt{8} / \pi<$ $h(x)^{1 / 2}$. Because $h$ is concave, $\left|f_{1}^{\prime}\right|+\left|f_{2}^{\prime}\right|=\left|h^{\prime}\right| \leq 2$ in $[a+1 / 2, b-1 / 2]$. Thus the assumption in part (b) implies that the Lipschitz constant of $\partial \Omega$ on $[x+1 / 2, x-1 / 2]$ is bounded by 2 . As in the appendix, the Harnack inequality and the Carleman lemma imply that $u(x, y)$ for any point $y$ in the middle third of the interval $f_{1}(x) \leq$ $y \leq f_{2}(x)$ majorizes values of $u$ in the full segment. And inequality (b) follows. Finally, (c) follows from Lemma A.6 applied to $w=u_{x}$ and $w=u_{y}$, together with Lemma A.5.

We now turn to global bounds. Recall from [J, (1.2) and (1.3)] the following bounds:

$$
\begin{gathered}
|\mu-\lambda|<C / L^{3}, \\
\lambda<\pi^{2}+C / L^{2} .
\end{gathered}
$$

Next, we define various subintervals of $[a, b]$. See Figure 2 .

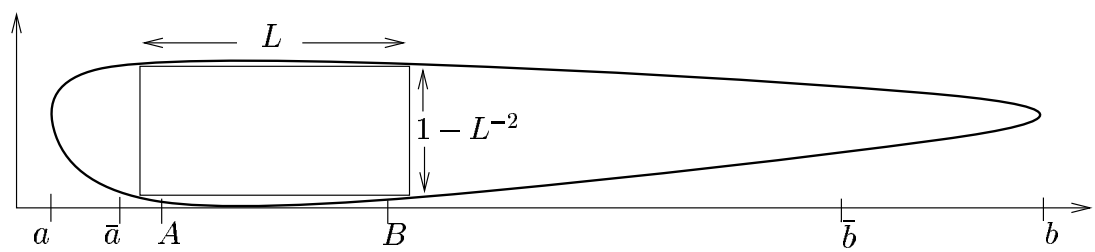

Figure 2: The length scale $L$

Definition 3.5. Let $0<\epsilon<1 / 3$. The middle (or $\epsilon$-middle) is the largest interval $I=[A, B]$ such that

$$
h(x)>1-1 / \epsilon L^{2} \text { for } A-\epsilon L \leq x \leq B+\epsilon L .
$$


We will sometimes display the dependence of the interval $I$ on $\epsilon$ with the notations $I(\epsilon), A(\epsilon)$, and $B(\epsilon)$. Note that if $0<\epsilon<\epsilon^{\prime}$, then $I\left(\epsilon^{\prime}\right) \subset I(\epsilon)$. More precisely,

$$
[A(\epsilon)-\epsilon L / 2, B(\epsilon)+\epsilon L / 2] \subset I(\epsilon / 2) .
$$

Let $I^{\prime}$ be the interval defined in Theorem 1.6. Then for sufficiently small $\epsilon>0$, $I^{\prime} \subset[A, B]$. We will prove Theorems 1.6 and 2.1 with $I^{\prime}$ replaced by the larger interval $[A, B]$, for arbitrary $\epsilon>0$ and constants depending on $\epsilon$.

Definition 3.6. Let $0<\epsilon_{0}<1 / 10$. The interval

$$
[\bar{a}, \bar{b}]
$$

is defined as the largest interval for which

$$
h(x) \geq 1-\epsilon_{0}, \quad\left|h^{\prime}(x)\right|<\epsilon_{0} \quad \text { for all } x \in[\bar{a}-2, \bar{b}+2] .
$$

The absolute constant $\epsilon_{0}$ will be chosen later, in the proof of Lemma 4.7. For $L$ large,

$$
a \leq \bar{a}-2<\bar{a}<A<B<\bar{b}<\bar{b}+2 \leq b .
$$

Also, $\max _{y} u$ and $\psi$ are comparable on $[\bar{a}, \bar{b}]$ by Lemma 3.2. Elementary reasoning with the slope of the concave function $h$ shows that

$$
L / 3<B-A<C_{\epsilon} L
$$

and

$$
\left|h^{\prime}\right|<C_{\epsilon} / L^{3} \text { on }[A-\epsilon L / 2, B+\epsilon L / 2] .
$$

We will show shortly that $\psi$ and $\phi$ are bounded away from 0 in the middle. By contrast, the interval $[\bar{a}, \bar{b}]$ can be much longer than $L$ and $\psi$ and $\phi$ will not be bounded below on such intervals as the following exponential decay estimate shows. For example, if $\Omega$ is a right triangle (or circular sector), then $N \approx L^{3}$ and $\bar{b}-\bar{a}$ is approximately $\epsilon_{0} N$.

Lemma 3.9. If $x_{1}$ is in the 1/3-middle, then for all $x \in[a, b]$

$$
\phi(x)+\psi(x) \leq C e^{-\left|x-x_{1}\right| / L} .
$$

In particular,

$$
\int_{a}^{b}(\psi(x)+\phi(x)) d x<C L .
$$

Proof. Define $\rho$ by

$$
\rho(x)=\int_{f_{1}(x)}^{f_{2}(x)} u(x, y)^{2} d y .
$$

Schwarz's inequality implies

$$
\psi(x)^{2} \leq \rho(x) .
$$




$$
\begin{aligned}
\rho^{\prime \prime} & =2 \int\left(u u_{x x}+u_{x}^{2}\right) d y=2 \int\left(-\lambda u^{2}-u u_{y y}+u_{x}^{2}\right) d y \\
& =2 \int\left(-\lambda u^{2}+u_{y}^{2}+u_{x}^{2}\right) d y \\
& \geq 2\left(\frac{\pi^{2}}{h^{2}}-\lambda\right) \int u^{2} d y+2 \int u_{x}^{2} \geq 2\left(\frac{\pi^{2}}{h^{2}}-\lambda\right) \rho .
\end{aligned}
$$

By definition of $L$ and convexity,

$$
h(x)<1-c\left|x-x_{1}\right| / L^{3} \quad \text { for all } \quad\left|x-x_{1}\right| \geq C L .
$$

This and the bound (3.4) give

$$
\rho^{\prime \prime}(x)>\frac{1}{L^{2}} \rho \quad \text { for all } \quad\left|x-x_{1}\right| \geq C L .
$$

For every $x, \rho(x) \leq \max u^{2}=1$. Let $x_{2}=\min \left(x_{1}+C L, b\right)$. The function

$$
R(x)=e^{-\left(x-x_{2}\right) / L}
$$

satisfies $R>0$ and $R^{\prime \prime}=R / L^{2}$. Therefore, by the generalized maximum principle (A.1), $\rho / R$ attains its maximum at an endpoint of the interval $\left[x_{2}, b\right]$. But $\rho\left(x_{2}\right) \leq$ $1=R\left(x_{2}\right)$ and $\rho(b)=0<R(b)$, so

$$
\rho(x) \leq R(x) \quad \text { for all } \quad x \in\left[x_{2}, b\right] .
$$

There is a similar argument on $\left[a, x_{1}-C L\right]$, and this gives the exponential decay for $\rho$. Since $\rho$ majorizes $\psi$, the bounds for $\psi$ are proved. The bounds for $\phi$ are proved in the same way as the bounds for $\rho$, since $\phi$ is positive in $(a, b), \phi(a)=\phi(b)=0$, and $\phi$ satisfies the differential equation $\phi^{\prime \prime}=\left(\pi^{2} / h(x)^{2}-\mu\right) \phi$, where $\mu$ has the same upper bound as $\lambda$.

Another upper bound on solutions is provided by the following lemma.

Lemma 3.12. There is an absolute constant $C$ such that

(a) $\max _{y} u(x, y) \leq C \min \{(x-a) / L,(b-x) / L\}$.

(b) If $h(x) \leq 1 / 2$, then $\max _{y} u(x, y) \leq C / L$.

Proof. We begin with the proof of the bound $(b-x) / L$. Estimate (3.4) implies that one can choose an absolute constant $c_{1}>0$ sufficiently small that

$$
R_{1}(s, t)=\sin \left(\pi(b-s) / c_{1} L\right) \sin (\pi t)
$$

satisfies $(\Delta+\lambda) R<0$. Because $\Omega$ is convex, a barrier on a semicircle (placed so that its flat side is tangent to the boundary) shows that $u$ vanishes at least to first order at the boundary, that is,

$$
u(s, t)<C \operatorname{dist}((s, t), \partial \Omega) .
$$

Since $0 \leq f_{1} \leq f_{2} \leq 1$, it follows that

$$
u\left(b-c_{1} L / 2, t\right) \leq C \sin (\pi t)=C R_{1}\left(b-c_{1} L / 2, t\right) .
$$

We will use the notation

$$
\Omega(\alpha, \beta)=\{(s, t) \in \Omega: \alpha<s<\beta\} .
$$

By the generalized maximum principle (A.1), $u / R_{1}$ achieves its maximum in $\Omega\left(b-c_{1} L / 2, b\right)$ on the boundary. In other words,

$$
u(x, y) \leq C R_{1}(x, y) \leq C \sin \left(\pi(b-x) / c_{1} L\right) .
$$


This proves the bound of order $(b-x) / L$ in part (a), and the corresponding bound of order $(x-a) / L$ follows by reflection $x \rightarrow-x$.

Next to prove part (b), fix $x$ such that $h(x) \leq 1 / 2$. Let $b_{1}=\sup \{x: h(x)>3 / 4\}$. We may assume, after a possible reflection, that $b_{1} \leq x$. The case $b-x \leq 1$ follows from part (a). So we may assume also that $b-x>1$. Then by concavity of $h$, $x-b_{1} \geq D / 3$ where $D=b-b_{1}$. Consider the infinite strip $I=\left[b_{1}, \infty\right) \times\left[y_{1}, y_{2}\right]$ with $y_{1}=f_{1}\left(b_{1}\right), y_{2}=f_{2}\left(b_{1}\right)$ and consider the function

$$
R(s, t)=e^{-\left(s-b_{1}\right) / 10} \sin \left(11 \pi\left(t-y_{0}\right) / 10\right) .
$$

The constant $y_{0}$ is chosen so that

$$
R\left(b_{1}, t\right)=\sin \left(11 \pi\left(t-y_{0}\right) / 10\right) \geq 1 / 100
$$

for all $t \in\left[y_{1}, y_{2}\right]$. This is possible because $y_{2}-y_{1} \leq 3 / 4$. By Lemma $3.1, \Omega\left(b_{1}, b\right) \subset$ $I$. Thus, since $u=0$ on $\partial \Omega$,

$$
u(s, t)<100 R(s, t) \max _{y} u\left(b_{1}, y\right) \quad \text { for all } \quad(s, t) \in \partial \Omega\left(b_{1}, b\right) .
$$

Finally, $R$ is a supersolution:

$$
(\Delta+\lambda) R=\left(-1.21 \pi^{2}+0.01+\lambda\right) R<0
$$

for sufficiently large $L$ by (3.4). Therefore, by the generalized maximum principle, (3.14) also holds on the interior of $\Omega\left(b_{1}, b\right)$. In particular,

$$
\max _{y} u(x, y) \leq 100 e^{-D / 30} \max _{y} u\left(b_{1}, y\right) .
$$

Finally, the estimate in part (a) implies

$$
\max _{y} u\left(b_{1}, y\right) \leq C D / L
$$

Combining this with (3.15) gives a bound of the form

$$
\max _{y} u(x, y) \leq C D e^{-D / 30} / L \leq C / L
$$

for suitable absolute constants $C$, changing from line to line.

A lower bound for the eigenfunction was proved in Proposition A and Lemma 2 of $[\mathrm{J}]$ and is restated here as follows.

Lemma 3.16. For any $\epsilon>0$ there exists $\delta>0$ such that if $x$ is in the $\epsilon$-middle, then

$$
\phi(x)>\delta,
$$

and such that under the additional assumption

$$
\operatorname{dist}((x, y), \partial \Omega)>\epsilon
$$

one has

$$
u(x, y) \geq \delta .
$$

We now prove a converse to Lemma 3.16 .

Lemma 3.17. For any $\delta>0$ there exists $\epsilon>0$ such that if $u(x, y) \geq \delta$, then $x$ is in the $\epsilon$-middle and

$$
\operatorname{dist}((x, y), \partial \Omega)>\epsilon
$$

and such that if $\phi(x) \geq \delta$, then $x$ is in the $\epsilon$-middle. 
Proof. We will first prove the claim for $u$. By the remark before (3.13) $u$ vanishes at least linearly at the boundary. It follows that there is an absolute constant $c$ such that $\operatorname{dist}((x, y), \partial \Omega) \geq c \delta$.

The conclusion that $x$ is in the $\epsilon$-middle will be proved in contrapositive form: For any $\delta>0$ there exists $\epsilon>0$ such that if $h(x-\epsilon L)<1-1 / \epsilon L^{2}$, then $u(x, y)<$ $\delta \max u$. (Using the symmetry $z \rightarrow-z$ the same argument is valid with $h(x-\epsilon L$ ) replaced by $h(x+\epsilon L)$.) Let $\epsilon \leq \delta^{2}$ and consider first the case $\delta \leq 1 / L$. Then the hypothesis becomes $h(x-\epsilon L)<0$, which must be interpreted to mean that $x-\epsilon L<a$. It follows from Lemma 3.12(a) that $\max _{y} u(x, y)<C(x-a) / L<C \epsilon=$ $C \delta^{2}$.

What remains is the case $\delta>1 / L$. Assume first that $h(x) \leq 1 / 2$. Then Lemma 3.12 (b) implies that $\max _{y} u(x, y)<C / L \leq C \delta$. So (replacing $\delta$ by a suitable multiple) we can assume that $h(x)>1 / 2$. Moreover, we can assume that $h(s) \geq 1 / 4$ for all $x-1<s<x+1$. To see this assume that $h(x+1)<1 / 4$. Then the convexity of $h$ implies $h(x+2)<0$, i.e., $b<x+2$. Lemma 3.12(a) implies that $\max _{y} u(x, y)<C(b-x) / L \leq 2 C / L \leq 2 C \delta$.

The preceding paragraph shows that one may assume that $h$ is uniformly bounded from below near $x$. By the Carleson lemma and Harnack inequality (as in Lemma $3.2) \max _{y} u(x, y)^{2} \leq \rho(x)$, where $\rho$ was defined in (3.10). Thus it suffices to estimate $\rho$.

Because $h$ is concave, it is monotone in two intervals of $[a, b]$. Consider first the case in which $h$ is increasing at $x-\epsilon L$. Then

$$
h(s)<1-1 / \epsilon L^{2} \text { for all } s \leq x-\epsilon L .
$$

Recall that $\rho$ defined in (3.10) satisfies

$$
\rho^{\prime \prime} \geq 2\left(\frac{\pi^{2}}{h^{2}}-\lambda\right) \rho .
$$

From (3.4), there is an absolute constant $C_{1}$ for which

$$
\rho^{\prime \prime}(s) \geq-C_{1}^{2} \rho / L^{2} \text { for all } s .
$$

Moreover (3.18) implies that for $\epsilon$ sufficiently small depending on the absolute constant in (3.4),

$$
\rho^{\prime \prime}(s) \geq\left(1 / \epsilon L^{2}\right) \rho \text { for all } s \leq x-\epsilon L .
$$

Let $\epsilon=\delta^{2}$. Define a supersolution $R(s)$ to dominate the subsolution $\rho$ as follows:

$$
\begin{gathered}
R(s)=\sin \left(C_{1}(s-x) / L+C_{1} \epsilon+C_{1} \delta\right), \quad s \geq x-\epsilon L, \\
R(s)=\left(\sin \left(C_{1} \delta\right)\right) e^{\delta+(s-x) / \delta L}, \quad s \leq x-\epsilon L .
\end{gathered}
$$

Note that $R$ is continuous at $s=x-\epsilon L$. Furthermore, since $\sin \left(C_{1} \delta\right)>C_{1} \delta \cos \left(C_{1} \delta\right)$, $R^{\prime}$ from the left is larger than $R^{\prime}$ from the right at $x=x-\epsilon L$. Thus $R^{\prime \prime}$ has a negative point mass at $s=x-\epsilon L$. At all other points the two inequalities for $\rho^{\prime \prime}$ imply that $R^{\prime \prime} / R \leq \rho^{\prime \prime} / \rho$. Finally we can make a comparison at endpoints. Namely, $\rho(a)=0$, but $R(a)>0$. And $R\left(x+\left(\pi / 2 C_{1}-\epsilon-\delta\right) L\right)=1$, and $\max \rho \leq 1$. In all, $R$ is a positive supersolution on the interval $\left[a, x+\left(\pi / 2 C_{1}-\epsilon-\delta\right) L\right]$. In particular, $\rho(x)<R(x)=\sin \left(C_{1} \epsilon+C_{1} \delta\right)$. Thus $\max _{y} u(x, y)^{2}<C \rho(x)<C \delta$ and the proof is complete if $\delta$ is replaced by a suitable multiple of $\delta^{2}$. (In the end we really used $\epsilon=\delta^{4}$.) 
Last of all we need to consider the case in which $h$ is decreasing at $x-\epsilon L$. But this case is easier than the other one. (3.18) is changed to

$$
h(s)<1-1 / \epsilon L^{2} \text { for all } s \geq x-\epsilon L,
$$

and $\rho$ is majorized by the barrier $R$ reflected through $x-\epsilon L$ :

$$
\rho(s) \leq R(-(s-(x-\epsilon L))+x-\epsilon L) .
$$

In particular, when $s=x, \rho(x) \leq R(x-2 \epsilon L) \leq \sin \left(C_{1} \delta\right)$.

The claim for $\phi$ can be proved in the same way since the differential inequality for $\rho$ is replaced by the equation $\phi^{\prime \prime}=\left(\pi^{2} / h^{2}-\mu\right) \phi$.

\section{$\S 4$. Estimates FOR $\sigma, u_{x}$ AND $\psi$}

We will now analyze

$$
\sigma=\left(\frac{d^{2}}{d x^{2}}-\frac{\pi^{2}}{h^{2}}+\mu\right) \psi
$$

A short calculation shows that

$$
\sigma=\int\left(2 e_{x} u_{x}+e_{x x} u\right) d y+(\mu-\lambda) \psi .
$$

Our goal is to give estimates for $\sigma$ in terms of $h^{\prime}, \psi$ and $\psi^{\prime}$ alone. The main problem will be to estimate $u_{x}$ in terms of $\psi^{\prime}$.

Lemma 4.1. $\left|h^{\prime} \psi\right| \leq C / L$ on $[a, b]$

Proof. Lemma 3.2(a) and Lemma 3.12(a) imply that

$$
\psi(x) \leq 5 \max _{y} u(x, y) \leq C \min (x-a, b-x) / L .
$$

But the concavity of $h$ implies that $\min (x-a, b-x) \leq 1 /\left|h^{\prime}(x)\right|$. This proves the lemma.

Lemma 4.2. $\left|\phi^{\prime}\right| \leq C / L$ on $[a, b]$.

Lemma 4.2 is proved in $[\mathrm{J}]$ (Lemma 2.4b).

Lemma 4.3. For any $\epsilon>0$ there exists $C$ depending only on $\epsilon$ such that if $[A, B]$ denotes the $\epsilon$-middle, then

(a) $\left|u_{x}(x, y)\right| \leq C / L$ for $A \leq x \leq B$,

(b) $u_{x}(x, y) \leq C \psi(x) / L$ for $B \leq x \leq \bar{b}$,

(c) $u_{x}(x, y) \geq-C \psi(x) / L$ for $\bar{a} \leq x \leq A$.

Proof. The key to the proof is that the convexity of the level sets of $u$ permits us to estimate their slope. (See Figure 3.) First consider the level set $u=0$, that is, $\partial \Omega$. There is an absolute constant such that

$$
\left|u_{x}\left(x, f_{2}(x)\right)\right|=\left|f_{2}^{\prime}(x) u_{y}\left(x, f_{2}(x)\right)\right| \leq\left|h^{\prime}(x)\right|\left|\nabla u\left(x, f_{2}(x)\right)\right| \leq C\left|h^{\prime}(x)\right| .
$$

The equality holds because the gradient is normal to the level set. The first inequality follows from Lemma 3.1 and the second from (A.4). Hence, (3.8) implies

$$
\left|u_{x}(x, y)\right| \leq C_{\epsilon} / L^{3}
$$

for all $(x, y) \in \partial \Omega$ for which $x \in[A-\epsilon L / 2, B+\epsilon L / 2]$, where $A=A(\epsilon)$ and $B=B(\epsilon)$ from Definition 3.5.

But boundary values of $u_{x}$ do not control the values on the interior of $\Omega$. Indeed the eigenfunction $u$ is zero on the boundary and nonzero inside. The main additional 


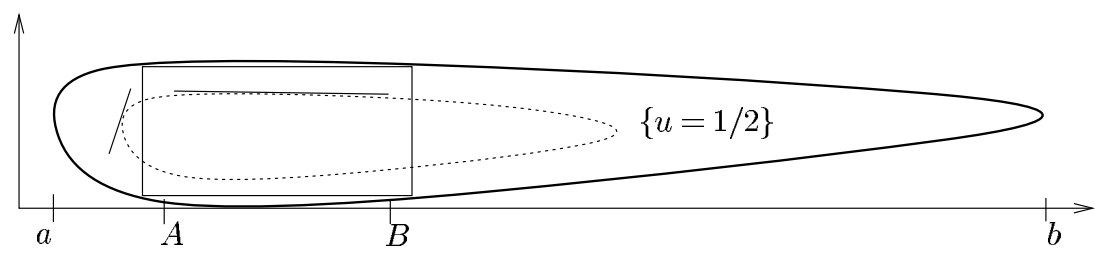

Figure 3: A level set of the eigenfunction $u$

element of the proof of (a) is to find another level set a fixed distance inside the domain for which the slope is bounded by $C / L$.

Choose $z_{1}$ so that for every $\left|z-z_{1}\right| \leq L / 2, h(z)>1-1 / L^{2}$. By Lemma 3.16 there is an absolute constant $\delta_{1}>0$ such that

$$
u\left(z_{1}, 1 / 2\right) \geq \delta_{1} \text {. }
$$

With the notations of Definition 3.5, let

$$
R(\epsilon)=\{(x, y): \epsilon<y<1-\epsilon, A(\epsilon)<x<B(\epsilon)\} .
$$

Denote

$$
\Omega_{\delta}=\{(x, y): u(x, y)>\delta\} .
$$

By Lemma 3.17 there is an absolute constant $\epsilon_{1}$ chosen depending on $\delta_{1}$ such that

$$
\Omega_{\delta_{1}} \subset R\left(\epsilon_{1}\right) .
$$

Let $\epsilon>0$ be the number in the hypothesis of Lemma 4.3. We may assume without loss of generality that $\epsilon<\epsilon_{1}$, so that $R\left(\epsilon_{1}\right) \subset R(\epsilon)$. Next, define an even larger rectangle $R^{\prime}$ by

$$
R^{\prime}=\{(x, y): \epsilon<y<1-\epsilon, A(\epsilon)-\epsilon L / 2<x<B(\epsilon)+\epsilon L / 2\} .
$$

For $L>2 / \epsilon$,

$$
\operatorname{dist}((x, y), \partial \Omega)>\epsilon / 2 \text { for every }(x, y) \in R^{\prime} .
$$

Thus by Lemma 3.16, there exists $\delta_{2}>0$, depending on $\epsilon$, such that $R^{\prime} \subset \Omega_{\delta_{2}}$. Finally, (the easy part of) Lemma 3.17 implies that there exists $\epsilon_{2}>0$ such that if $(x, y) \in \Omega_{\delta_{2}}$, then

$$
\epsilon_{2}<y<1-\epsilon_{2}
$$

Consider any point $(x, y) \in \partial \Omega_{\delta_{2}}$ for which $A-\epsilon L / 4 \leq x \leq A+\epsilon L / 4$. Because $\Omega_{\delta_{2}}$ is convex and contains $R^{\prime}$, the slope of the tangent line to $\partial \Omega_{\delta_{2}}$ at $(x, y)$ has absolute value less than $4 / L$. As before, when we estimated $u_{x}$ on $\partial \Omega$, it follows that

$$
\left|u_{x}(x, y)\right| \leq 4|\nabla u(x, y)| / L \leq C / L .
$$

In summary, $\left|u_{x}\right| \leq C / L$ on the four curves forming the upper and lower boundaries of $\Omega$ and $\Omega_{\delta_{2}}$ for $A-\epsilon L / 4 \leq x \leq B+\epsilon L / 4$. Also we have the bound $\left|u_{x}\right| \leq C$ everywhere which follows from (A.4). We now deduce that

$$
\left|u_{x}\right| \leq C / L \text { for } A \leq x \leq B
$$


using barriers on rectangles containing the three regions between these boundaries: First, consider the middle strip $\epsilon_{2}<y<1-\epsilon_{2}$. The function

$$
F(x, y)=\left(1 / \epsilon_{2} L\right) \cosh (x / \sqrt{L}) \sin \left(\pi+\epsilon_{2}\right) y
$$

satisfies

$$
F(x, y) \geq(1 / L) \cosh (x / \sqrt{L}) \geq 1 / L \text { for } \epsilon_{2} \leq y \leq 1-\epsilon_{2} .
$$

Moreover, when $x= \pm \epsilon L / 4$,

$$
F(x, y) \geq(1 / L) e^{\epsilon \sqrt{L} / 4}>>1 .
$$

Moreover, for sufficiently large $L$, depending on $\epsilon_{2}$, the eigenvalue of $F$ is larger than $\lambda$ :

$$
\left(\pi+\epsilon_{2}\right)^{2}-1 / L>\pi^{2}+100 / L^{2}>\lambda
$$

by (3.4). Therefore, $F$ is a supersolution. For each point $x \in[A, B]$ translate $F$ by $x$. Then $F$ majorizes $\pm u_{x}$ on the boundary of $\Omega_{\delta_{2}}(x-\epsilon L / 4, x+\epsilon L / 4)$. Hence by the generalized maximum principle, (A.1), it majorizes it inside. The value of $F$ at $x=0$, the center of the rectangle, is at most $1 / \epsilon_{2} L$. Therefore, we have $\left|u_{x}(x, y)\right| \leq C / L$ for all $(x, y) \in \Omega_{\delta_{2}}$ such that $A \leq x \leq B$. The other two regions of $\Omega$ in $A \leq x \leq B$ are much narrower, so the same barrier, translated up or down in $y$, provides the same bound. This concludes the bounds in Lemma 4.3(a).

Next consider any $(x, y) \in \Omega$ for which $x \geq B$. Then $u(x, y)<\delta_{1}$. The convexity of the level sets of $u$ implies that the half-plane

$$
H=\{(z, w):(z-x, w-y) \cdot \nabla u(x, y)>0\}
$$

contains $\Omega_{\delta_{1}}$. In particular, $\left(z_{1}, 1 / 2\right) \in \Omega_{\delta_{1}}$, so that

$$
\left(z_{1}-x\right) u_{x}(x, y)+\left(\frac{1}{2}-y\right) u_{y}(x, y)>0 .
$$

But $z_{1}-x \leq-L / 2$. Therefore, if $x \leq \bar{b}$, Lemma 3.2 implies

$$
L u_{x}(x, y) / 2<\left|u_{y}(x, y)\right| \leq C \psi(x) .
$$

The proof for $x \leq A$ is similar.

Denote

$$
F_{I}(x)=\int_{x-1}^{x+1} F(t) d t \quad \text { and } \quad S=\left|h^{\prime}\right|_{I} .
$$

Lemma 4.4. (a) $|\sigma| \leq C\left(S\left|\psi^{\prime}\right|_{I}+\left(S / L+S^{2}+\left|h^{\prime \prime}\right|+L^{-3}\right) \psi\right)$ on $[\bar{a}, \bar{b}]$, for an absolute constant $C$.

(b) $|\sigma| \leq C\left(L^{-3}+\left|h^{\prime \prime}\right|\right)$ on $[A, B]$, for a constant $C$ depending on $\epsilon$, where $A=A(\epsilon), B=B(\epsilon)$.

(c) In particular, $|\sigma|_{I} \leq C L^{-3}$ on $[A, B]$, for a constant $C$ depending on $\epsilon$.

We note in particular that the constant in part (a) does not depend on $\epsilon_{0}$ of Definition 3.6. This is obvious because a smaller $\epsilon_{0}$ gives a smaller interval $[\bar{a}, \bar{b}]$.

Proof. Suppose that we are in the region $A \leq x \leq B$. Then $\psi>c>0$ and according to Lemma 4.3, $\left|u_{x}\right| \leq 1 / L$. We also have $\left|\bar{e}_{x}\right| \leq C\left|h^{\prime}\right|$ and $\left|e_{x x}\right| \leq C\left(\left|h^{\prime \prime}\right|+\left|h^{\prime}\right|^{2}\right)$. Finally, $|\mu-\lambda| \leq C L^{-3}$ from (3.3). Therefore, in this range the bound is valid without recourse to the term involving $\psi^{\prime}$. 
Next, consider $B \leq x \leq \bar{b}$. Then Lemma 4.3 says that

$$
u_{x} \leq C \psi / L \text {. }
$$

Note that

$$
\psi^{\prime}=\int\left(e_{x} u+e u_{x}\right) d y
$$

so that, using Lemma 3.2(b),

$$
\left|\int e u_{x} d y\right|=\left|-\int e_{x} u d y+\psi^{\prime}\right| \leq C\left|h^{\prime}\right| \psi+\left|\psi^{\prime}\right| .
$$

Therefore,

$$
\begin{aligned}
0 \leq \int e\left(u_{x}\right)_{-} d y & =-\int e u_{x} d y+\int e\left(u_{x}\right)_{+} d y \\
& \leq-\int e u_{x} d y+C \psi / L \\
& \leq C\left(\left|\psi^{\prime}\right|+\left|h^{\prime}\right| \psi+\psi / L\right) .
\end{aligned}
$$

It follows that

$$
\int e\left|u_{x}\right| d y \leq C\left(\left|\psi^{\prime}\right|+\left|h^{\prime}\right| \psi+\psi / L\right) .
$$

The boundary values of $u_{x}$ are bounded by $\left|h^{\prime}\right||\nabla u|$. This in turn is bounded by $C\left|h^{\prime}\right| \psi$, by Lemma A.5 and Lemma 3.2. Lemma A.6 implies

$$
\sup _{y}\left|u_{x}(x, y)\right| \leq C \sup _{|z-x|<1 / 2}\left(\left|\psi^{\prime}(z)\right|+\left|h^{\prime}(z)\right| \psi(z)+\psi(z) / L\right) .
$$

Because $h$ is concave, $\left|h^{\prime}\right|_{I}$ dominates the maximum of $\left|h^{\prime}\right|$ on the central portion of the interval. Also the Harnack inequality and Carleman inequalities as in Lemma 3.2 imply that $\psi_{I}$ is comparable to $\psi$ on $[\bar{a}, \bar{b}]$. Therefore

$$
\left|u_{x}\right| \leq C\left(\left|\psi^{\prime}\right|_{I}+\left|h^{\prime}\right|_{I} \psi+\psi / L\right) \text {. }
$$

This completes the proof of Lemma 4.4(a). Part (b) is a consequence of part (a) and the fact that $S$ is bounded by $C / L^{3}$ on $[A, B]$ and $\left|\psi^{\prime}\right| \leq C$ from Lemma 3.2. To prove part (c), integrate the bound in part (b) and use the fact that $\left|h^{\prime}\right| \leq C / L^{3}$ on $[A, B]$ to bound the integral of $h^{\prime \prime}$ on a unit interval.

Lemma 4.5. There is a constant $C\left(\epsilon_{0}\right)$ depending on the constant $\epsilon_{0}$ in Definition 3.6 used to define $\bar{a}$ and $\bar{b}$ such that

(a) $\int_{a}^{\bar{a}+1}+\int_{\bar{b}-1}^{b}(\psi+\phi) \leq C\left(\epsilon_{0}\right) / L$,

(b) $|\psi(x)|+|\phi(x)|+\left|\psi^{\prime}(x)\right|+\left|\phi^{\prime}(x)\right| \leq C\left(\epsilon_{0}\right) / L$ for all $x$ such that $|x-\bar{a}| \leq 1$ or $|x-\bar{b}| \leq 1$.

Proof. The definition (3.6) of $\bar{b}$ allows for two possibilities, one in which $h^{\prime}(\bar{b}+2) \leq$ $-\epsilon_{0}$ (if $h^{\prime}$ is discontinuous the limit from the right must satisfy this inequality) and one in which $h(\bar{b}+2)=1-\epsilon_{0}$. In the first case, concavity of $h$ implies $b-(\bar{b}+2) \leq 1 / \epsilon_{0}$ or $b-(\bar{b}-2) \leq 4+1 / \epsilon_{0}$. Therefore, by Lemma 3.2(a) and Lemma $3.12(\mathrm{a})$,

$$
\psi(x) \leq \max _{y} u(x, y) \leq\left(4+1 / \epsilon_{0}\right) C / L \quad \text { for all } \bar{b}-2 \leq x \leq b .
$$


It follows that

$$
\int_{\bar{b}-2}^{b} \psi(x) d x \leq\left(4+1 / \epsilon_{0}\right)^{2} C / L
$$

Now consider the second case, $h(\bar{b}+2)=1-\epsilon_{0}$. First, if $\bar{b}$ is within distance $4 / \epsilon_{0}$ of $b$, then the estimates for $\psi$ follow from Lemma 3.12(a). Otherwise, we have

$$
h(\bar{b}-1)<1-\epsilon_{0} / 4
$$

by concavity. In that case, the estimate

$$
u(\bar{b}-1, y)<C\left(\epsilon_{0}\right) / L
$$

follows exactly as in Lemma $3.12(\mathrm{~b})$, with $1 / 2$ replaced by $1-\epsilon_{0} / 4$. This gives the upper bound for $\psi$. The integral bound for $\psi$ then follows from the exponential decay estimate

$$
\rho(x) \leq C \rho(\bar{b}-1) e^{-c(x-\bar{b})},
$$

which is proved exactly as in the proof of Lemma 3.9, only with (3.11) replaced by $\rho^{\prime \prime}>c^{2} \rho$ where $c=c\left(\epsilon_{0}\right)>0$.

Next, let us bound $\psi^{\prime}$. For $x$ in the range $|x-\bar{b}| \leq 1, h(x) \geq 1-\epsilon_{0} \geq 1 / 2$ and $\left|h^{\prime}(x)\right| \leq \epsilon_{0} \leq 1$ and hence $\left|e_{x}\right| \leq C$. Furthermore, by Lemma 3.2(c), $\max _{y}\left|u_{x}(x, y)\right| \leq \max _{y} u(x, y)$, which is bounded as above by $C\left(\epsilon_{0}\right) / L$. It follows that

$$
\left|\psi^{\prime}(x)\right|=\left|\int e_{x} u d y+\int e u_{x} d y\right| \leq C\left(\epsilon_{0}\right) / L \quad \text { for all } \quad|x-\bar{b}| \leq 1 .
$$

This argument shows that $\left|\psi^{\prime}(x)\right| \leq \max _{y} u(x, y)$ for any $x$ for which $h\left(x^{\prime}\right)>1 / 2$ for all $\left|x^{\prime}-x\right|<1$. The bounds involving $\bar{a}$ are proved similarly.

The bound for $\phi^{\prime}$ in part (b) is a special case of Lemma 4.2. The bound for $\phi$ follows from an argument similar to the one above, but much simpler. The maximum principle (A.1) applies in one variable as well as several variables. The function $\phi$ satisfies

$$
(\mathcal{L}+\mu) \phi=\left(\frac{d^{2}}{d x^{2}}-V_{1}\right) \phi=0
$$

with $V_{1}=\pi^{2} / h(x)^{2}-\mu$. $V_{1}(x) \geq-100 / L^{2}$, so $(\mathcal{L}+\mu) g<0$ for $g(x)=\sin (C(b-x) / L)$ and $C>10$. This function $g$ is the supersolution that gives the estimate of Lemma $3.2(\mathrm{a})$. The exponential decay is proved in the interval where $V_{1}(x)>\delta_{0}^{2}$ using appropriate multiples of the supersolution $e^{-\delta_{0}(x-\bar{b})}$.

Lemma 4.7. There are absolute constants $\epsilon_{0}$ and $C$ so that with $\bar{a}, \bar{b}$ defined with this $\epsilon_{0}$,

(a) $\left|\psi^{\prime}\right| \leq C / L$ on $[\bar{a}, \bar{b}]$,

(b) $\int_{\bar{a}}^{\bar{b}}|\sigma| \leq C / L$.

Proof. Recall that $h>1-\epsilon_{0}$ and $\left|h^{\prime}\right|<\epsilon_{0}$ on $[\bar{a}-1, \bar{b}+1]$. The function $\psi$ satisfies

$$
\psi^{\prime \prime}=V_{1} \psi+\sigma \quad \text { where } V_{1}=\frac{\pi^{2}}{h^{2}}-\mu \geq-100 / L^{2} .
$$


Furthermore, $V_{1} \geq 0$, except on an interval of length comparable to $L$. Also, $0 \leq \psi \leq 1$. Therefore, for any $\alpha<\beta$,

$$
\int_{\alpha}^{\beta} V_{1} \psi \geq-C / L
$$

Because $\psi(x)$ is comparable to $\max _{y} u(x, y) h(x)^{1 / 2}$, Lemma 3.17 implies that there exists $\epsilon>0$ defining $A$ and $B$ so that $\psi$ attains its maximum at a point $\bar{x} \in[A, B]$. Fix this $\epsilon$ throughout this proof. The constant $\epsilon_{0}$ will be chosen at the end only, so we have to keep track of the dependence of various constants on $\epsilon_{0}$.

Recall that for sufficiently large $L$, depending on $\epsilon_{0},[A, B] \subset[\bar{a}, \bar{b}]$. For $\bar{x} \leq x \leq$ $\bar{b},(4.8)$ implies

$$
\psi^{\prime}(x)=\int_{\bar{x}}^{x} V_{1} \psi+\sigma \geq-C L^{-1}-\int_{\bar{x}}^{x}|\sigma| .
$$

Also, (4.8) and Lemma 4.5(b) imply

$$
\psi^{\prime}(x)=\psi^{\prime}(\bar{b})-\int_{x}^{\bar{b}}\left(V_{1} \psi+\sigma\right) \leq C\left(\epsilon_{0}\right) L^{-1}+\int_{x}^{\bar{b}}|\sigma| .
$$

Denote

$$
M=\max _{\bar{b} \geq x \geq \bar{x}}\left|\psi^{\prime}(x)\right| .
$$

Actually, $\psi^{\prime}$ has jump discontinuities where the monotone function $h^{\prime}$ is discontinuous. Those points correspond to places where $\sigma$ has a point mass. The pointwise equations written here can be modified by taking suitable left and right limits. What we just proved implies that

$$
M \leq C\left(\epsilon_{0}\right) / L+\int_{\bar{x}}^{\bar{b}}|\sigma|,
$$

in which the integral in $|\sigma|$ is interpreted as including any point mass at the endpoints.

Now we estimate the $|\sigma|$ integral using Lemma 4.4:

$$
\int_{\bar{x}}^{\bar{b}}|\sigma| \leq C\left(\int_{\bar{x}}^{\bar{b}} S\left|\psi^{\prime}\right|_{I}+\int_{\bar{x}}^{\bar{b}}\left(S / L+S^{2}+\left|h^{\prime \prime}\right|+1 / L^{3}\right) \psi\right) .
$$

Lemma A.5 implies $\left|\psi^{\prime}\right| \leq C$ on $[\bar{a}-1, \bar{b}-1]$. To bound the first term, use the definition of $M$, Lemma 4.5 (b) and (3.8) to get

$$
\begin{aligned}
\int_{\bar{x}}^{\bar{b}} S\left|\psi^{\prime}\right|_{I} & \leq \int_{\bar{x}}^{\bar{b}} S\left|\psi^{\prime}\right|+\int_{\bar{x}-1}^{\bar{x}} S\left|\psi^{\prime}\right|+\int_{\bar{b}-1}^{\bar{b}} S\left|\psi^{\prime}\right| \\
& \leq \int_{\bar{x}}^{\bar{b}} S M d x+C / L^{3}+C\left(\epsilon_{0}\right) / L .
\end{aligned}
$$

Since $h$ is concave, $\left|h^{\prime \prime}\right|=-h^{\prime \prime}$, so integration by parts and Lemma 4.5(b) yield

$$
\begin{aligned}
-\int_{\bar{x}}^{\bar{b}} h^{\prime \prime}(x) \psi(x) d x & =\int_{\bar{x}}^{\bar{b}} h^{\prime}(x) \psi^{\prime}(x) d x+\psi(\bar{x}) h^{\prime}(\bar{x})-\psi(\bar{b}) h^{\prime}(\bar{b}) \\
& \leq \int_{\bar{x}}^{\bar{b}}\left|h^{\prime}(x)\right| M d x+C / L^{3}+C\left(\epsilon_{0}\right) / L .
\end{aligned}
$$


Next,

$$
\int_{\bar{x}}^{\bar{b}} S(x)^{2} \psi(x) d x \leq C \int_{\bar{x}-1}^{\bar{b}+1} h^{\prime}(x)^{2} \psi(x) d x .
$$

One can replace the average $S$ with the value $\left|h^{\prime}(x)\right|$ because Harnack's inequality implies $\psi(x)$ changes by at most a constant factor on unit intervals in $[\bar{a}-1, \bar{b}+1]$. Note that the constant does not depend on $\epsilon_{0}$ because as $\epsilon_{0}$ gets smaller the interval $[\bar{a}, \bar{b}]$ gets smaller. Lemma 4.1 now gives

$$
\int_{\bar{x}-1}^{\bar{b}+1} h^{\prime}(x)^{2} \psi(x) d x \leq(C / L) \int\left|h^{\prime}\right| \leq C / L .
$$

The remaining terms are bounded using $\psi \leq 1$ and Lemma 3.9,

$$
\int_{\bar{x}}^{\bar{b}}\left(S / L+C / L^{3}\right) \psi \leq C \int_{a}^{b}\left|h^{\prime}(x)\right| d x / L+C L / L^{3} \leq C / L .
$$

Assembling all the terms gives the following bound on the integral in $|\sigma|$ :

$$
\int_{\bar{x}}^{\bar{b}}|\sigma| \leq C M \int_{\bar{x}-1}^{\bar{b}+1}\left|h^{\prime}\right|+C\left(\epsilon_{0}\right) / L .
$$

The constant $C$ multiplying the first factor is independent of $\epsilon_{0}$. Since

$$
\int_{\bar{a}-1}^{\bar{b}+1}\left|h^{\prime}\right| \leq 2 \epsilon_{0}
$$

combining this with (4.9) gives

$$
M \leq 2 C M \epsilon_{0}+C\left(\epsilon_{0}\right) / L .
$$

Choose $\epsilon_{0}$ to be a sufficiently small absolute constant such that $2 C M \epsilon_{0}<1 / 2$. Then subtract to find $M \leq C / L$ for some absolute constant $C$. This bound and (4.11) give the bound on the integral of $|\sigma|$. There is a symmetric argument for the interval $[\bar{a}, \bar{x}]$, so this concludes the proof of Lemma $4.7(\mathrm{a})$ and (b).

\section{$\S 5$. Proof of Bounds on $u-\psi e$ of Theorem 2.1(A)}

Let $v=u-\psi e$; then we want to prove

$$
|v(x, y)| \leq C / L^{3} \text { for } x \in[A, B] .
$$

As we remarked earlier (after Definition 3.5) $I^{\prime} \subset[A, B]$, whenever $\epsilon$ is sufficiently small. So this estimate covers the range stated in Theorem 2.1(a).

Define

$$
\tau(x)=\int_{f_{1}(x)}^{f_{2}(x)} v(x, y)^{2} d y .
$$

Let

$$
E=e \sigma+2 e_{x} \psi^{\prime}+e_{x x} \psi
$$

and

$$
\beta(x)=2\left(\int E(x, y)^{2} d y\right)^{1 / 2}
$$


One calculates (see [GJ], section 4; the calculation there is for the second eigenfunction, but it carries over literally)

$$
(\Delta+\lambda) v=-E,
$$

and from this

$$
\tau^{\prime \prime} \geq \tau-\beta \sqrt{\tau} .
$$

On $[A-\epsilon L / 2, B+\epsilon L / 2]$ we have $\left|h^{\prime}\right| \leq C / L^{3}$ and $\left|\sigma_{I}\right| \leq C / L^{3}$ by applying Lemma $4.4(\mathrm{c})$ to the interval $[A(\epsilon / 2), B(\epsilon / 2)]$, so

$$
\beta_{I} \leq C L^{-3} \quad \text { on } \quad[A-\epsilon L / 2, B+\epsilon L / 2] .
$$

Lemma 5.3 (see [GJ], Lemma 3). Suppose that $\tau$ is an absolutely continuous function satisfying $(5.1)$ on $[0,1]$ and

$$
\tau(0) \geq\left(10 \int_{0}^{1} \beta\right)^{2}, \quad \tau^{\prime}(0) \geq 0 .
$$

Then there exists $x \in[0,1]$ satisfying

$$
\tau(x)>(6 / 5) \tau(0), \quad \tau^{\prime}(x) \geq 0 .
$$

We now prove

$$
\tau(x) \leq C_{0} L^{-6} \quad \text { on } \quad[A-1, B+1]
$$

as in the proof of Lemma 4 in [GJ]; here $C_{0}=(10 C)^{2}$ with $C$ the constant in (5.2): We will argue by contradiction. Fix a point $x \in[A-1, B+1]$ and assume $\tau(x)>C_{0} L^{-6}$. Without loss of generality we may assume $\tau^{\prime}(x) \geq 0$, otherwise we reflect about the point $x$. Apply Lemma 5.3 with 0 replaced by $x$, to obtain a new point $x_{1}$. Apply the lemma again with 0 replaced by $x_{1}$, and iterate this step $\epsilon L / 2-1$ times. This keeps us always within the interval $[A-\epsilon L / 2, B+\epsilon L / 2]$. If $x^{\prime}$ is the point obtained in the last iteration, we have

$$
\tau\left(x^{\prime}\right) \geq(6 / 5)^{\epsilon L / 2-1} \tau(x) \geq(6 / 5)^{\epsilon L / 2-1} C_{0} L^{-6}>2
$$

for large $L$. But $\tau\left(x^{\prime}\right) \leq 2$ for all $x^{\prime}$, the desired contradiction.

So we have proved (5.4), i.e. we know that the $L^{2}$ norm of $v$ over the $y$-interval satisfies the desired bound, uniformly in $x \in[A, B]$. In section 5 of [GJ] it is proved, using integral bounds on the Green's function of the Dirichlet Laplacian on a unit width domain, that these imply pointwise bounds of the form

$$
|v(x, y)| \leq e(x, y)(|\log e(x, y)|+1) L^{-3} .
$$

(The factor $\left|x-x_{0}\right| / L$ in front of the log term in Lemma 5 of [GJ] is missing here since it comes from an estimate on the size of the second eigenfunction near its nodal line. But the size of the first eigenfunction is of unit size on $[A, B]$. Also, the factor $W$ is replaced by the weaker estimate $L^{-3}$ from (5.4) in the present paper.) In particular, $|v(x, y)| \leq C L^{-3}$ for $x \in[A, B]$ and this implies Theorem 2.1(a). 


\section{§6. Proof of Bounds on $\psi-\phi / \sqrt{2}$ of Theorem 2.1(в)}

Let $0 \leq \chi \leq 1$ be a cutoff function which equals one on $[\bar{a}+1, \bar{b}-1]$, whose support is contained in $[\bar{a}, \bar{b}]$, and such that $\chi^{\prime}$ and $\chi^{\prime \prime}$ are bounded by, say, 10 . The support of $\chi^{\prime}$ is contained in $[\bar{a}, \bar{a}+1] \cup[\bar{b}-1, \bar{b}]$. Choose any point $x_{1}$ in the $\epsilon$-middle, $[A, B]$, and set

$$
c_{0}=\psi\left(x_{1}\right) / \phi\left(x_{1}\right) .
$$

By Lemma 3.16, $c_{0}$ is bounded away from zero and infinity. Denote

$$
\begin{gathered}
\eta_{0}(x)=\psi(x)-c_{0} \phi(x), \\
\eta(x)=\chi(x) \eta_{0}(x) .
\end{gathered}
$$

Thus $\eta\left(x_{1}\right)=0$ and

$$
(\mathcal{L}+\mu) \eta=\chi \sigma+2 \chi^{\prime} \eta_{0}^{\prime}+\chi^{\prime \prime} \eta_{0}
$$

In order to prove Theorem 2.1(b), we will first show

$$
|\eta(x)| \leq C / L \quad \text { for } \quad x \in[A, B],
$$

and then

$$
\left|c_{0}-1 / \sqrt{2}\right|<C / L \text {. }
$$

We want to express $\eta$ using the solution $\phi$ of $(\mathcal{L}+\mu) \phi=0$, vanishing at $b$, and a second solution, vanishing at $x_{1}$, which can be constructed as follows:

$$
\tilde{\phi}(x)=\phi(x) \int_{x_{1}}^{x} \phi(t)^{-2} d t .
$$

Then $(\mathcal{L}+\mu) \tilde{\phi}=0$ and $\tilde{\phi}\left(x_{1}\right)=0$. Because $\mathcal{L}$ does not have a first-order term, the Wronskian $W=\tilde{\phi}^{\prime}(x) \phi(x)-\tilde{\phi}(x) \phi^{\prime}(x)$ is constant. Note that

$$
\tilde{\phi}^{\prime}\left(x_{1}\right)=\phi\left(x_{1}\right)^{-1} \text {. }
$$

Therefore, $W=1$. The method of variation of parameters (with the boundary conditions $\eta\left(x_{1}\right)=\eta(b)=0$ ) gives

$$
\eta(x)=-\phi(x) \int_{x_{1}}^{x} \tilde{\phi}\left(\chi \sigma+2 \chi^{\prime} \eta_{0}^{\prime}+\chi^{\prime \prime} \eta_{0}\right)-\tilde{\phi}(x) \int_{x}^{\bar{b}} \phi\left(\chi \sigma+2 \chi^{\prime} \eta_{0}^{\prime}+\chi^{\prime \prime} \eta_{0}\right) .
$$

Here the second integral should really go up to $b$, but the integrand is zero on $[\bar{b}, b]$.

To deduce (6.1) from this, let $x \in[A, B]$. In this range $\phi(x)>c>0$, and $\left|x-x_{1}\right| \leq C L$, so that

$$
\tilde{\phi}(x) \leq C L \phi(x) .
$$

Thus the formula for $\eta$ implies

$$
|\eta(x)| \leq C L \phi(x)\left(\int_{x_{1}}^{\bar{b}} \phi|\sigma|+\int_{\bar{b}-1}^{\bar{b}} \phi\left(\left|\eta_{0}^{\prime}\right|+\eta_{0}\right)\right) .
$$

By Lemma 4.5(b), the second integral is bounded by $C / L^{2}$. Therefore, (6.1) will follow from

$$
\int_{x_{1}}^{\bar{b}} \phi|\sigma| \leq C / L^{2}
$$


In other words, using Lemma 4.4(a), we need to show

$$
\int_{x_{1}}^{\bar{b}}\left(\phi\left|\psi^{\prime}\right|_{I} S+\phi \psi S / L+\phi \psi S^{2}+\phi \psi\left|h^{\prime \prime}\right|+\phi \psi / L^{3}\right) d x \leq C / L^{2}
$$

Below we will prove the essential new ingredient

$$
\int_{\bar{a}}^{\bar{b}} S(\psi+\phi) \leq C / L
$$

Assuming this for the moment, we prove (6.3): The term $\int \phi\left|\psi^{\prime}\right|_{I} S$ is handled using $\left|\psi^{\prime}\right|<C / L$ (Lemma 4.7(a)) and (6.4), the term $\int \phi \psi S / L$ using $\phi \leq 1$ and (6.4), the term $\int \phi \psi S^{2}$ using $S \psi<C / L$ (Lemma $4.1 ; S$ is controlled by $\left|h^{\prime}\right|$ on $[\bar{a}, \bar{b}]$ ) and (6.4), the term $\int \phi \psi / L^{3}$ using $\phi \leq 1$ and the exponential decay of $\psi$ (Lemma 3.9), and finally the term $\int \phi \psi h^{\prime \prime}=-\int \phi \psi\left|h^{\prime \prime}\right|$ using integration by parts:

$$
\int_{x_{1}}^{\bar{b}} \phi \psi h^{\prime \prime} d x=\left.\phi \psi h^{\prime}\right|_{x_{1}} ^{\bar{b}}-\int_{x_{1}}^{\bar{b}}(\phi \psi)^{\prime} h^{\prime} d x
$$

the boundary terms are bounded by $C / L^{2}$ since $\left|h^{\prime}\right|<C / L^{3}$ in the middle and $\phi, \psi$ are bounded by $C / L$ at $\bar{b}$. The remaining integral is handled in the same way as the first term in (6.3).

It only remains to prove (6.4). As before, we can replace $S$ by $\left|h^{\prime}\right|$. We will first show

$$
\int_{\bar{a}}^{\bar{b}}\left|h^{\prime}\right| \psi \leq C / L
$$

This inequality is far from obvious since the interval $[\bar{a}, \bar{b}]$ can be much longer than $C L$ and the only uniform bound on the integrand is $C / L$ from Lemma 4.1. One way to deal with this would be a dyadic decomposition on the interval, with careful estimates on the sizes of $h^{\prime}$ and $\psi$ separately on each piece. Instead we use the equation

$$
\left(-\frac{d^{2}}{d x^{2}}+V_{1}\right) \psi=\sigma, \quad V_{1}=\frac{\pi^{2}}{h^{2}}-\lambda,
$$

and a type of energy

$$
\mathcal{E}=\left(\psi^{\prime}\right)^{2}-V_{1} \psi^{2}
$$

The point is that the integrand in $(6.5)$ is closely related to $\mathcal{E}^{\prime}$ :

$$
\mathcal{E}^{\prime}=2 \psi^{\prime}\left(\psi^{\prime \prime}-V_{1} \psi\right)-V_{1}^{\prime} \psi^{2}=2 \sigma \psi^{\prime}-V_{1}^{\prime} \psi^{2}
$$

and

$$
V_{1}^{\prime}=-2 \pi^{2} h^{-3} h^{\prime} \approx-h^{\prime}
$$

Choose $z_{1} \in[\bar{a}, \bar{b}]$ with $h^{\prime} \leq 0$ on $\left[z_{1}, \bar{b}\right]$. Then $V_{1}^{\prime} \geq 0$ and

$$
\begin{aligned}
\int_{z_{1}}^{\bar{b}} V_{1}^{\prime} \psi^{2} & =\mathcal{E}\left(z_{1}\right)-\mathcal{E}(\bar{b})-2 \int_{z_{1}}^{\bar{b}} \sigma \psi^{\prime} \\
& \leq \psi^{\prime}\left(z_{1}\right)^{2}+V_{1}(\bar{b}) \psi(\bar{b})^{2}+2 \int_{z_{1}}^{\bar{b}}\left|\sigma \psi^{\prime}\right| \leq C / L^{2} .
\end{aligned}
$$


The last bound here comes from Lemma 4.5(b) and Lemma 4.7. Since $V_{1}^{\prime}$ is comparable to $\left|h^{\prime}\right|$ in $\left[z_{1}, \bar{b}\right]$, we have, by the Cauchy-Schwarz inequality

$$
\int_{z_{1}}^{\bar{b}}\left|h^{\prime}\right| \psi \leq C \int_{z_{1}}^{\bar{b}} V_{1}^{\prime} \psi \leq C\left(\int_{z_{1}}^{\bar{b}} \psi^{2} V_{1}^{\prime}\right)^{1 / 2}\left(\int_{z_{1}}^{\bar{b}} V_{1}^{\prime}\right)^{1 / 2} \leq C / L .
$$

To estimate the integral over an interval on which $h^{\prime} \geq 0$, we proceed analogously. Finally, the same estimate works for $\phi$, only it is simpler because the term $\sigma$ is missing. This completes the proof of (6.1).

We now prove (6.2). Assume $u$ takes its maximum 1 at $\left(x_{0}, y_{0}\right)$, and $\phi$ takes its maximum 1 at $x_{1}$. By Lemma 3.17, there is an $\epsilon$ so that both $x_{0}$ and $x_{1}$ lie in the $\epsilon$-middle. On $x \in[A, B]$ and any $y$ we have, by Theorem 2.1(a),

$$
\psi(x) \leq\left(u(x, y)+C L^{-3}\right) / e(x, y) .
$$

Evaluating at the center $y=\left(f_{1}(x)+f_{2}(x)\right) / 2$, we get, using $u \leq 1$,

$$
\psi(x) \leq \sqrt{h(x) / 2}+C L^{-3} \text {. }
$$

On the other hand,

$$
\psi\left(x_{0}\right) \geq\left(1-C L^{-3}\right) / e\left(x_{0}, y_{0}\right) \geq \sqrt{h\left(x_{0}\right) / 2}-C L^{-3} .
$$

In both of these estimates, $h$ can be replaced by 1 since $h=1-O\left(L^{-3}\right)$ in the middle.

Therefore, we get at $x_{0}$, using (6.1),

$$
c_{0} \geq c_{0} \phi\left(x_{0}\right) \geq \psi\left(x_{0}\right)-C / L \geq \sqrt{1 / 2}-C / L,
$$

and at $x_{1}$

$$
c_{0}=c_{0} \phi\left(x_{1}\right) \leq \psi\left(x_{1}\right)+C / L \leq \sqrt{1 / 2}+C / L .
$$

This proves (6.2) and thereby concludes the proof of Theorem 2.1 .

\section{$\S 7$. Location of the MaXimum}

Proof of (2.10). As before, we write

$$
V_{1}=\pi^{2} / h^{2}-\mu \text {. }
$$

By Lemma 3.17 we can choose $\epsilon$ so small that $\max \{\phi(A), \phi(B)\}<1 / 2$ where $A=A(2 \epsilon), B=B(2 \epsilon)$. By Lemma 4.2 we then have $\min \left(B-x_{1}, x_{1}-A\right) \geq c L, c>0$. Further, by Lemma 3.16 we have $\phi \geq c_{0}>0$ on $[A-\epsilon L, B+\epsilon L] \subset[A(\epsilon), B(\epsilon)]$. We rescale to unit lengths by setting

$$
\begin{gathered}
\alpha=\left(A-x_{1}\right) / L, \quad \beta=\left(B-x_{1}\right) / L, \\
V(x)=L^{2} V_{1}\left(L x+x_{1}\right), \quad p(x)=\phi\left(L x+x_{1}\right) .
\end{gathered}
$$

Then (2.10) follows from:

Lemma 7.1. Let $V$ be a convex function on $[\alpha-\epsilon, \beta+\epsilon]$, where $\alpha<0<\beta, \epsilon>0$, and such that

$$
V \leq C
$$

there. Assume that the function $p$ satisfies the equation

$$
p^{\prime \prime}=V p \quad \text { on }[\alpha-\epsilon, \beta+\epsilon],
$$


and the following bounds:

$$
\begin{gathered}
p(0)=\max p=1, \\
\max \{p(\alpha), p(\beta)\}<1 / 2, \\
p \geq c_{0}>0 \quad \text { on }[\alpha-\epsilon, \beta+\epsilon],
\end{gathered}
$$

and that $p$ is monotone on each of the intervals $[\alpha-\epsilon, 0],[0, \beta+\epsilon]$. Then

$$
\left|p^{\prime}(x)\right| \geq c|x|
$$

where the constant $c>0$ is bounded away from zero if $|\alpha|, \beta, C$ are bounded above and $\alpha, \beta, \epsilon, c_{0}$ are bounded away from zero.

Proof. In this proof, all constants $c_{1}, c_{2}, \ldots$ will be positive and bounded away from 0 in the same way as is stated for $c$. Since $p^{\prime \prime}$ changes sign at most once on each of the intervals $[\alpha, 0],[0, \beta]$, it is enough to prove $(7.3)$ on some neighborhood $\left[-c_{1}, c_{1}\right]$ of zero, and at $\alpha$ and $\beta$.

First, we show

$$
V(0) \leq-c_{2}
$$

We may assume, possibly after a reflection, that $V^{\prime}(0) \geq 0$. By convexity, $V$ is then increasing on $[0, \beta]$. From $(7.2)$ and $p^{\prime}(0)=0$ we conclude

$$
\int_{0}^{\beta}(\beta-x) V(x) p(x) d x=p(\beta)-p(0)<-1 / 2
$$

and from this $\beta^{2} c_{0} V(0)<-1 / 2$. This proves (7.4).

Now convexity of $V$ and $V \leq C$ imply for some $c_{1}<\min (|\alpha|, \beta)$

$$
V(x) \leq-c_{2} / 2 \text { for } x \in\left[-c_{1}, c_{1}\right]
$$

and thus

$$
\left|p^{\prime}(x)\right|=\left|\int_{0}^{x} V p\right| \geq|x| c_{2} c_{0} / 2 .
$$

It remains to show $p^{\prime}(\alpha) \geq c_{3}$. The bound at $\beta$ will be analogous. We distinguish the cases $V(\alpha) \leq 0$ and $V(\alpha)>0$.

If $V(\alpha) \leq 0$, then $V \leq 0$ on $[\alpha, 0]$ by convexity, so $p^{\prime}$ is decreasing there and

$$
p^{\prime}(\alpha) \geq p^{\prime}\left(-c_{1}\right) \geq c_{0} c_{1} c_{2} / 2 .
$$

Finally, if $V(\alpha)>0$, then $V \geq c_{4}$ on $[\alpha-\epsilon, \alpha-\epsilon / 2]$ by convexity and (7.4). Also, $p^{\prime}$ is increasing on $[\alpha-\epsilon / 2, \alpha]$, so

$$
p^{\prime}(\alpha) \geq p^{\prime}(\alpha-\epsilon / 2)=p^{\prime}(\alpha-\epsilon)+\int_{\alpha-\epsilon}^{\alpha-\epsilon / 2} V p \geq 0+c_{4} c_{0} \epsilon / 2 .
$$

This concludes the proof of the lemma, and thus of (2.10).

Proof of (2.9). Let $\bar{x}$ and $x$ belong to $[A, B]$. Recall that

$$
\phi^{\prime}(x)=\phi^{\prime}(\bar{x})+\int_{\bar{x}}^{x} V_{1} \phi
$$


and

$$
\psi^{\prime}(x)=\psi^{\prime}(\bar{x})+\int_{\bar{x}}^{x}\left(V_{1} \psi+\sigma\right) .
$$

Write $\eta=\psi-\phi / \sqrt{2}$. In $[A, B],\left|V_{1}\right| \leq C / L^{2}, \sigma_{I} \leq C / L^{3}$, and $|\eta| \leq C / L$ by Theorem 2.1(b) so that

$$
\left|\eta^{\prime}(x)-\eta^{\prime}(\bar{x})\right| \leq \int_{A}^{B} C L^{-3} \leq C / L^{2} .
$$

Integrating this last inequality in $x$ over $[A, B]$, with $\bar{x}$ fixed, we find

$$
\left|\eta(B)-\eta(A)-\eta^{\prime}(\bar{x})(B-A)\right| \leq C / L .
$$

On the other hand,

$$
|\eta(B)|+|\eta(A)| \leq C / L
$$

Therefore,

$$
\left|\eta^{\prime}(\bar{x})(B-A)\right| \leq C / L .
$$

Since $B-A \geq c L$ we find that

$$
\left|\eta^{\prime}(\bar{x})\right| \leq C / L^{2}
$$

for every $\bar{x} \in[A, B]$. This proves $(2.9)$.

Now let us prove Theorem 1.3. By Lemma 3.17, there is an $\epsilon>0$ so that both $x_{0}$ and $x_{1}$ lie in the $\epsilon$-middle. Also, assume $\epsilon$ is chosen small enough for (2.10) to be valid. Note that $\epsilon$ can be chosen as an absolute constant, since it depends only on the previous choice of various absolute constants. Recall that $\left|h^{\prime}\right| \leq C / L^{3}$ in the middle and $h=1+O\left(1 / L^{2}\right)$. Therefore, $\psi(x) e(x, 1 / 2)$ and its first derivative satisfies the same estimate as $\sqrt{2} \psi(x)$ in its comparison with $\phi$. Combining (2.9) and (2.10) we have absolute constants $c>0$ and $C$ such that

$$
(d / d x)(\psi(x) e(x, 1 / 2)) \leq-c\left(x-x_{1}\right) / L^{2}+C / L^{2}
$$

for all $x$ such that $x_{1}<x \leq B$. Note that the largest value of $u$ is 1 , so that, by Theorem 2.1(a), the largest value of $\psi e$ is at most $1+O\left(1 / L^{3}\right)$. The upper bound on the derivative of $\psi e$ implies that for some absolute constant $C, \psi(x) e(x, 1 / 2) \leq$ $1-1 / L^{2}$ whenever $x_{1}+C<x \leq B$. Since the midpoint of the segment $\left[f_{1}(x), f_{2}(x)\right]$ is within $O\left(1 / L^{2}\right)$ of $1 / 2, e(x, y) \leq\left(1+O\left(1 / L^{4}\right)\right) e(x, 1 / 2)$. Thus Theorem 2.1(a) implies that $u(x, y)<1$ for all $x_{1}+C<x \leq B$. Since we know that $x_{0} \leq B$, we get $x_{0} \leq x_{1}+C$. The lower bound is similar.

\section{Appendix: Potential theory of eigenfunctions in CONVEx Domains}

The generalized maximum principle can be stated as follows ([PW, Theorem 10 p. 73]).

Theorem A.1. Let $D$ be a bounded, open subset of $\mathbf{R}^{2}$. Suppose that $g$ is a strictly positive continuous function on $\bar{D}$. Suppose that $(\Delta+\lambda) g \leq 0$ in $D$. Let $u$ be continuous in $\bar{D}$ and satisfy $(\Delta+\lambda) u \geq 0$ in $D$. If $u \leq g$ on $\partial D$, then

$$
u \leq g \text { on } D \text {. }
$$

(The proof is an application of the usual maximum principle to the function $u / g$.) 
Remark A.2. The theorem is also valid in $\mathbf{R}^{n}$ with the same proof and when $\lambda$ is a continuous function rather than a constant. This is used in the body of the paper only in the case of ordinary differential equations $(n=1)$.

Inequality (3.4) is proved by using as a test function the eigenfunction in an inscribed rectangle with sides $L$ and $1-2 / L^{2}$. The eigenvalue is

$$
\pi^{2}\left(L^{-2}+\left(1-2 / L^{2}\right)^{-2}\right),
$$

which is less than 50 if $L \geq 2$. Therefore, if $L \geq 2$,

$$
\lambda \leq 50 .
$$

We use this upper bound to prove linear decay of the eigenfunction at the boundary.

Proposition A.4. Suppose that $\lambda \leq 50$. Let $u$ solve $(\Delta+\lambda) u=0$ in a convex domain $\Omega$. Let $z_{0} \in \partial \Omega$ and let $B_{s}$ denote the disk of radius $s$ around $z_{0}$. If $u=0$ on $B_{1 / 2} \cap \partial \Omega$, then

$$
|u(z)| \leq C\left|z-z_{0}\right| \sup _{B_{1 / 4} \cap \Omega}|u|, \text { for all } z \in B_{1 / 8} \cap \Omega .
$$

In particular, if $u \geq 0$ in $\Omega$, then

$$
\left|\nabla u\left(z_{0}\right)\right| \leq C \sup _{B_{1 / 4} \cap \Omega}|u| .
$$

(Note that in this case $\nabla u$ is defined almost everywhere with respect to arclength on the boundary by Dahlberg's theorem.)

Proof. After a rotation, we may assume that a tangent line to $\partial \Omega$ at $z_{0}$ is parallel to the $x$-axis in $z=x+i y$-space and $\Omega$ lies above the $x$-axis. Translate so that $z_{0}=i \epsilon$ for some fixed $\epsilon, 0<\epsilon<1 / 100$. To construct a barrier function, denote

$$
\beta=\arg \left(\frac{1+z}{1-z}\right)
$$

where $z=x+i y$ identifies $(x, y)$ with a complex variable. (Note that $\beta$ is harmonic because it is the imaginary part of $\log (1+z) /(1-z)$.) The barrier is

$$
g=\pi \beta-\beta^{2} .
$$

To show that $g$ is a supersolution, note that $\beta(z)=0$ for $z$ real and $-1<z<1$. Furthermore, $\beta(z)=\pi / 2$ when $|z|=1$ in $\operatorname{Im} z>0$. Therefore, $g$ attains its maximum in the semicircle $\{z: \operatorname{Im} z \geq 0,|z| \leq 1\}$ on the circular boundary where $\beta$ is $\pi / 2$ and $g$ is $\pi^{2} / 4$. An easy computation using the Cauchy-Riemann equations shows

$$
|\nabla \beta|=\left|\frac{2}{1-z^{2}}\right| \geq 2 .
$$

Using $\Delta \beta=0$, one computes that $g$ is a supersolution:

$$
\Delta g=-2|\nabla \beta|^{2} \leq-8 \leq-32 g / \pi^{2} \leq-3 g .
$$

For any constant $r$, the function $g(z / r)$ is a subsolution for $\Delta+3 / r^{2}$ on the semicircle of radius $r$. Fix $r=1 / 5$. The bound $\lambda \leq 50$ then implies $\Delta g(z / 5)<-75 g(z / 5)<$ $-\lambda g(z / 5)$. Let $D=B_{1 / 5}(0) \cap \Omega$. The function $g(z / 5)$ is continuous and positive 
on $\bar{D}$. Furthermore, $g=\pi^{2} / 4>1$ on $\bar{\Omega} \cap \partial B_{1 / 5}(0) \subset B_{1 / 4}\left(z_{0}\right)$. Therefore, since $u$ is zero on the remaining part of the boundary of $D$,

$$
\max _{\partial D} u / g \leq \sup _{B_{1 / 4}\left(z_{0}\right) \cap \Omega} u .
$$

It follows from Theorem A.1 that either $u / g \leq 0$ on $\bar{D}$ or

$$
\max _{\bar{D}} u / g \leq \sup _{B_{1 / 4}\left(z_{0}\right) \cap \Omega} u
$$

Combined with the similar bound for $-u$, this gives

$$
\max _{\bar{D}}|u| / g \leq \sup _{B_{1 / 4}\left(z_{0}\right) \cap \Omega}|u| \text {. }
$$

To get the estimate of Proposition A.4, one takes the limit as $\epsilon$ tends to zero.

Lemma A.5. Suppose the planar convex domain $\Omega$ is normalized as in the body of the paper. Suppose that $x_{0} \in[a+1, b-1]$ and $h\left(x_{0}\right)>1 / 10$. There is an absolute constant $C$ such that if $u>0$ is the first eigenfunction on $\Omega$, then

$$
\max \left\{|\nabla u(x, y)|:(x, y) \in \partial \Omega,\left|x-x_{0}\right| \leq 1 / 2\right\} \leq C \max _{y} u\left(x_{0}, y\right) .
$$

Furthermore,

$$
\max \left\{|\nabla u(x, y)| \cdot \operatorname{dist}((x, y), \partial \Omega):\left|x-x_{0}\right| \leq 1 / 2\right\} \leq C \max _{y} u\left(x_{0}, y\right) .
$$

Note: The second bound can be improved using the first bound and Lemma A.6 below. See Lemma 3.2(c).

Proof. The assumptions on $h$ and $x_{0}$ imply that there is a uniformly bounded Lipschitz constant for the domain $\Omega \cap\left\{\left|x-x_{0}\right|<3 / 4\right\}$. Harnack's inequality and the Carleson Lemma (see [J], Propositions 3.3 and 3.4) imply that

$$
\max \left\{u(x, y):\left|x-x_{0}\right| \leq 5 / 8\right\} \leq C \max _{y} u\left(x_{0}, y\right)
$$

Then the first inequality for the gradient at the boundary follows from Proposition A.4. The second follows from the well-known fact ${ }^{3}$ that if $(\Delta+\lambda) u=0$ in $B_{r}$, then

$$
r \max _{B_{r / 2}}|\nabla u| \leq C \max _{B_{r}}|u|
$$

for $r<c \lambda^{-1 / 2}$.

Lemma A.6. Let $w$ satisfy $(\Delta+\lambda) w=0$ in $\Omega$. Define $\bar{a}, \bar{b}$ as in Definition 3.6. For $\bar{a} \leq x_{0} \leq \bar{b}$,

$$
\begin{aligned}
\sup _{y}\left|w\left(x_{0}, y\right)\right| \leq C & \iint_{\left|x-x_{0}\right| \leq 1 / 2}|w(x, y)| e(x, y) d x d y \\
& +C \sup \left\{|w(x, y)|:(x, y) \in \partial \Omega,\left|x-x_{0}\right| \leq 1 / 2\right\} .
\end{aligned}
$$

\footnotetext{
${ }^{3}$ This fact can be reduced to the case $r=1$ by dilation. In that case it follows from the explicit representation of solutions to the eigenvalue equation or standard interior elliptic regularity estimates.
} 
Proof. Clearly, the number $1 / 2$ can be replaced by any positive constant. For ease of notation, we will prove the lemma with $1 / 2$ replaced by 1 . Recall that for $x \in[\bar{a}, \bar{b}], e(x, y)$ is comparable to distance to the boundary. So $e(x, y)$ is bounded below by an absolute constant if $\operatorname{dist}((x, y), \partial \Omega)>1 / 10$. Provided the point $\left(x_{0}, y\right)$ is at least a fixed distance from the boundary, interior regularity for $\Delta+\lambda$ implies that $w\left(x_{0}, y\right)$ is bounded by the integral on the right side.

To obtain estimates up to the boundary, define

$$
c=\min _{\left|x-x_{0}\right|<1 / 10} f_{2}(x)-1 / 10 .
$$

Because $\left|f_{2}^{\prime}(x)\right| \leq\left|h^{\prime}(x)\right|<1 / 10$, the points

$$
z_{1}=\left(x_{0}-1, c\right) \quad \text { and } \quad z_{2}=\left(x_{0}+1, c\right)
$$

both belong to $\Omega$. For $1 / 3 \leq t \leq 2 / 3$, define (Figure 4 )

$$
D_{t}=\left\{z=(x, y) \in \Omega:\left|x-x_{0}\right|<1, y>c,\left|z-z_{1}\right|>t,\left|z-z_{2}\right|>t\right\} .
$$

Define the four "sides" of $D_{t}$ as follows:

$$
\partial D_{t}=\Gamma_{1} \cup \Gamma_{2} \cup \Gamma_{3} \cup \Gamma_{4}
$$

where

$$
\begin{aligned}
& \Gamma_{1}=\left\{(x, c): x_{0}-1+t \leq x \leq x+0+1-t\right\}, \\
& \Gamma_{2}=\left\{z \in \partial D_{t}:\left|z-z_{1}\right|=t\right\}, \\
& \Gamma_{3}=\left\{z \in \partial D_{t}:\left|z-z_{2}\right|=t\right\}, \\
& \Gamma_{4}=\left(\partial D_{t}\right) \cap \partial \Omega .
\end{aligned}
$$

The crucial geometric feature of these curves is that because $z_{1} \in \Omega, \Gamma_{2}$ meets $\Gamma_{4}$ in an acute interior angle, and similarly, $\Gamma_{3}$ meets $\Gamma_{4}$ in an acute interior angle. This will show that the Poisson kernel has at least linear decay new $\Gamma_{4}$, which permits the use of the weight factor $e(x, y)$ in the integral in Lemma A.6. The linear decay rate is not special - the Poisson kernel associated to foliations by curves that meet the boundary at even more narrow angles have arbitrarily fast algebraic decay rates. Thus the same bounds would be true with any power of $e(x, y)$. But the first power is what arises in our application.

Assume $z_{0}=\left(x_{0}, y_{0}\right)$ belongs to $D_{t}$. We will make uniform estimates independent of the choice of $y_{0}$ in the interval $c \leq y_{0} \leq f_{2}\left(x_{0}\right)$. Because $D_{t}$ is a narrow domain (width less than $4 / 10$ and length less than 2) one can choose a suitable

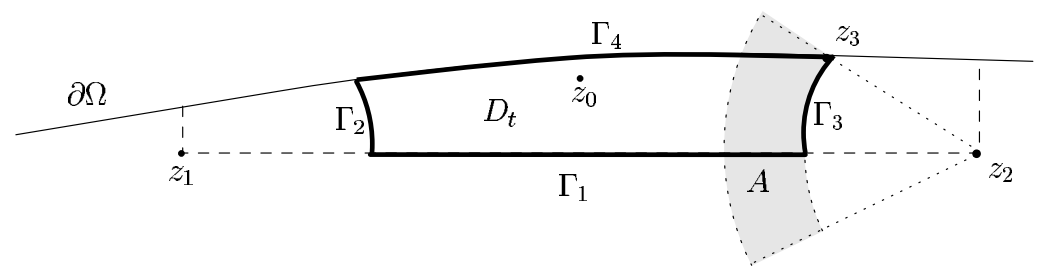

Figure 4: Lemma A.6 
translate of the function

$$
\sin (\pi x / 2) \sin 2 \pi y
$$

denoted by $g$ so that $g$ has a uniform positive lower bound on $\bar{D}_{t}$ independent of $t, 1 / 3 \leq t \leq 2 / 3$. Because $\lambda<4 \pi^{2}, g$ is a supersolution for $\Delta+\lambda$. From this one obtains uniqueness of the solution for the Dirichlet problem for $\Delta+\lambda$ in $D_{t}$ (see $[\mathrm{PW}]$, Theorem 11, p. 73). Existence then follows from the Fredholm alternative since the Dirichlet Laplacian is selfadjoint and one can use the compact operator whose integral kernel is the standard Green's function of the Laplacian on $D_{t}$ as an approximate inverse for $\Delta+\lambda$, so $\Delta+\lambda$ is selfadjoint and Fredholm. Thus we have Green's function $G$, depending on $t$, so that

$$
h(z)=\int_{D_{t}} G(z, \zeta) f(\zeta) d \zeta
$$

satisfies $(\Delta+\lambda) h=f$ in $D_{t}$ and $h=0$ on $\partial D_{t}$. By standard arguments the homogeneous boundary problem is then also solvable, so we also have the Poisson kernel $P$, that is,

$$
w(z)=\int_{\partial D_{t}} P(z, \zeta) w(\zeta) d \sigma(\zeta)
$$

for every solution $w$ to $(\Delta+\lambda) w=0$ in $D_{t}$ with integrable boundary values. The generalized maximum principle (A.1) implies that $P \geq 0$. The supersolution $g$ satisfies

$$
g(z) \geq \int_{\partial D_{t}} P(z, \zeta) g(\zeta) d \sigma(\zeta)
$$

and comparable upper and lower bounds for $g$ therefore imply

$$
\sup _{z \in D_{t}} \int_{\partial D_{t}} P(z, \zeta) d \sigma(\zeta) \leq C
$$

uniformly in $t, 1 / 3 \leq t \leq 2 / 3$.

To deduce uniform decay estimates for $P$ near the boundary, we use the representation

$$
P(z, \zeta)=(d / d n) G(z, \zeta)
$$

where $d / d n$ denotes the normal derivative at $\zeta$ on the boundary and estimate $G$. First, we claim that

$$
|G(z, \zeta)| \leq C
$$

uniformly for $|z-\zeta|>1 / 100$ and uniformly in $t$. Let $J$ be the radial Bessel potential solving $(\Delta+\lambda) J=\delta$, the Dirac delta, in $\mathbf{R}^{2}$. If $z$ is a fixed distance from the boundary of $D_{t}$, the formula

$$
G(z, \zeta)=J(\zeta-z)-\int_{\partial D_{t}} P(z, s) J(s-z) d \sigma(s)
$$

proves (A.8) because $J(\zeta-z)$ is bounded above on $\partial D_{t}$. Moreover, if $z$ is near the boundary one can use the method of images. In place of $J(\zeta-z)$ use $J(\zeta-z)-$ $J\left(\zeta-z^{*}\right)$ where $z^{*}$ is in the complement of $D_{t}$ and $\operatorname{dist}\left(z, \partial D_{t}\right) \approx \operatorname{dist}\left(z^{*}, \partial D_{t}\right)$. Because $|J(z)|-c \log |z|$ is bounded for $|z|<2, J(\zeta-z)-J\left(\zeta-z^{*}\right)$ is uniformly bounded for $\zeta \in \partial D_{t}$ as $z$ tends to $\partial D_{t}$. 
Formula (A.8) and the semicircular barrier used in the proof of (3.13) shows that

$$
\left|G\left(z_{0}, \zeta\right)\right| \leq C \operatorname{dist}\left(\zeta, \Gamma_{4}\right)
$$

provided $\left|z_{0}-\zeta\right|>1 / 100$. Next, we wish to prove quadratic decay of $G$ near the corner $\Gamma_{3} \cap \Gamma_{4}$. We claim that

$$
G\left(z_{0}, \zeta\right) \leq C \operatorname{dist}\left(\zeta, \Gamma_{3}\right) \operatorname{dist}\left(\zeta, \Gamma_{4}\right)
$$

for all $\zeta$ near $z_{3}=\Gamma_{3} \cap \Gamma_{4}$. This can be proved using an explicit barrier in polar coordinates with origin at $z_{2}$. Consider such polar coordinates $(r, \theta)$ and let $\theta_{0}$ be the angle of the ray from $z_{2}$ to $z_{3}$. The annulus

$$
A=\left\{(r, \theta): \theta_{0}<\theta<\theta_{0}+\pi / 3, t<r<t+1 / 10\right\}
$$

contains a $1 / 10$ distance neighborhood of $\Gamma_{3}$ in $D_{t}$. The focus here is on the rightangle corner $\theta=\theta_{0}, r=t$ of the annulus. This containment property is where we use the fact that $\Gamma_{3}$ meets $\Gamma_{4}$ at an acute angle. Define the function

$$
g_{\epsilon}=(f(r)+\epsilon) \sin \left(\theta-\theta_{0}+\epsilon\right) \quad(0<\epsilon<1 / 20)
$$

where $f(r)=(r-t)-5(r-t)^{2}$. One computes that $g_{\epsilon}$ is a supersolution for $\Delta+\lambda$ in the annulus $A$. Indeed,

$$
(\Delta+\lambda) g_{\epsilon}=\left[f^{\prime \prime}+\frac{1}{r} f^{\prime}+\left(\frac{-1}{r^{2}}+\lambda\right)(f+\epsilon)\right] \sin \left(\theta-\theta_{0}+\epsilon\right)<0 .
$$

This is because $f^{\prime \prime}(r)=-10, f^{\prime}(r) \leq 1$ and $f(r)+\epsilon \leq 1 / 10$ for $t \leq r \leq t+1 / 10$. For $L \geq 2, \lambda \leq 50$, so that the sum in parentheses is less than $-10+3+50 / 10<0$. (Recall the standing assumption that $1 / 3 \leq t \leq 2 / 3$ implies that $r \geq 1 / 3$.) Next since $g_{\epsilon}>0$ on the annulus, $f \geq 1 / 20$ on $r=t+1 / 10$, and $\sin \left(\theta-\theta_{0}+\epsilon\right) \geq\left(\theta-\theta_{0}\right) / 2$, (A.9) implies that a fixed multiple of $g_{\epsilon}$ majorizes $G$ on the boundary of the annulus $A$. Theorem A.1 implies that $g_{\epsilon}$ majorizes $G$ on the interior of $A$. But both the factor $f$ and the factor $\sin \left(\theta-\theta_{0}\right)$ vanish at $r=t, \theta=\theta_{0}$, so, letting $\epsilon$ tend to zero, the bound (A.10) follows.

Taking the normal derivative of $G$ at $\zeta$ we find

$$
P\left(z_{0}, \zeta\right) \leq C \operatorname{dist}\left(\zeta, \Gamma_{4}\right) \quad \text { for all } \quad \zeta \in \Gamma_{3} .
$$

This bound, the symmetric one on $\Gamma_{2}$, and (A.7) imply

$$
\left|w\left(z_{0}\right)\right| \leq C \int_{\Gamma_{2} \cup \Gamma_{3}}|w(\zeta)| \operatorname{dist}\left(\zeta, \Gamma_{4}\right) d \sigma(\zeta)+C \sup _{\Gamma_{1} \cup \Gamma_{4}}|w(\zeta)|
$$

with constants independent of the coordinate $y$ in $z_{0}=\left(x_{0}, y\right)$ and also independent of $t, 1 / 3 \leq t \leq 2 / 3$. Thus one can integrate the right hand side in $t$. The curves $\Gamma_{2}$ and $\Gamma_{3}$ sweep out a two-dimensional subset of $\Omega$ contained in $\left|x-x_{0}\right| \leq 1$. Moreover, the interior bounds mentioned at the beginning of the proof show that the maximum of $w$ in $\Gamma_{1}$ is bounded by the double integral as well. This concludes the proof of the lemma.

\section{REFERENCES}

[B1] C. Borell, Hitting probabilities of killed Brownian motion; a study on geometric regularity, Ann. Scient. École Norm. Sup. 17 (1984), 451-467. MR 86h:60157

[B2] C. Borell, Greenian potentials and concavity, Math. Ann. 272 (1985), 155-160. MR 87h:31007

[BL] H. J. Brascamp and E. Lieb, On extensions of the Brunn-Minkowski and Prekopa-Leindler theorems, including inequalities for log concave functions, and with an application to a diffusion equation, J. Funct. Anal. 22 (1976), 366-389. MR 56:8774 
[CL] E. A. Coddington and N. Levinson, Theory of Ordinary Differential Equations, Robert E. Krieger, Malabar, Florida, 1984, 1955 edition published by McGraw-Hill. MR 16:1022b

[D] T. A. Driscoll, Eigenmodes of isospectral drums, SIAM Rev. 39 (1997), 1-17. CMP 97:09

[GJ] D. Grieser and D. Jerison, Asymptotics of the first nodal line of a convex domain, Inventiones Math. 125 (1996), 197-219. MR 97d:35033

[J] D. Jerison, The diameter of the first nodal line of a convex domain, Annals of Math. 141 (1995), 1-33. MR 95k:35148

[J1] D. Jerison, Locating the first nodal line in the Neumann problem, Trans. A. M. S. (to appear).

[KP] C. E. Kenig and J. Pipher, The h-path distribution of the lifetime of conditioned Brownian motion for non-smooth domains, Prob. Th. and Rel. Fields 82 (1989), 615-624. MR 91a:60211

[K] P. Kröger, On the ground state eigenfunction of a convex domain, Potential Anal. 5 (1996), 103-108. MR 96j:35038

[PW] M. H. Protter and H. F. Weinberger, Maximum Principles in Differential Equations, Springer Verlag, New York, 1984. MR 86f:35034

[PW1] M. H. Protter and H. F. Weinberger, An optimal Poincaré inequality for convex domains, Arch. Rational Mech. Anal. 5 (1960), 286-292.

[S] R. G. Smits, Spectral gaps and rates to equilibrium for diffusions in convex domains, Michigan Math. J. 43 (1996), 141-157. MR 97d:35037

Humboldt Universität Berlin, Institut für Mathematik, Unter den Linden 6, 10099 Berlin, Germany

E-mail address: grieser@mathematik.hu-berlin.de

Department of Mathematics, Massachusetts Institute of Technology, Cambridge, MASSACHUSETTS 02139

E-mail address: jerison@math.mit.edu 